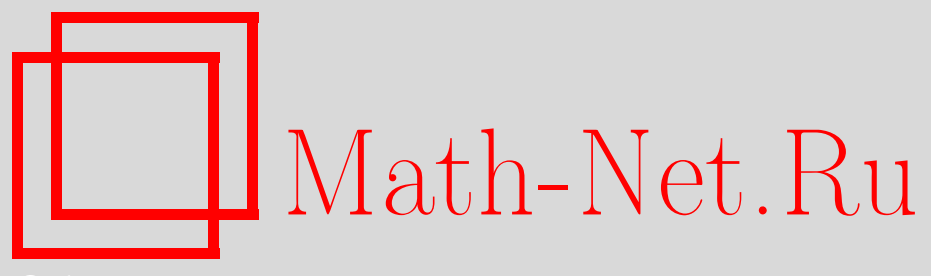

А. А. Толстоногов, Сходимость по Моско интегральных функционалов и ее приложения, Матем. сб., 2009, том 200, номер 3, 119-146

DOI: https://doi.org/10.4213/sm5007

Использование Общероссийского математического портала Math-Net.Ru подразумевает, что вы прочитали и согласны с пользовательским соглашением http://www . mathnet.ru/rus/agreement

Параметры загрузки:

IP : 3.85 .183 .62

26 апреля 2023 г., 15:32:43

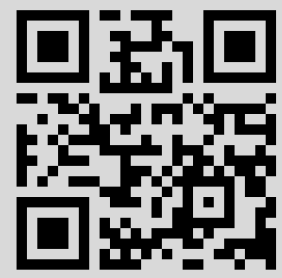




\title{
А. А. Толстоногов \\ Сходимость по Моско интегральных функционалов и ее приложения
}

\begin{abstract}
Изучаются вопросы сходимости по Моско интегральных функционалов, определенных на пространстве интегрируемых с квадратом функций со значениями в гильбертовом пространстве. Интегрантами у этих функционалов являются зависящие от времени собственные выпуклые полунепрерывные снизу функции, определенные на гильбертовом пространстве. Полученные результаты применяются к изучению зависимости от параметра решений эволюционных уравнений с зависящими от времени субдифференциальными операторами. В качестве примера рассмотрено параболическое включение, в правую часть которого входит сумма $p$-лапласиана и субдифференциала индикаторной функции зависящего от времени выпуклого замкнутого множества. Исследована сходимость решений этого включения при $p \rightarrow+\infty$.
\end{abstract}

Библиография: 20 названий.

Ключевые слова: сходимость по Моско, интегральные функционалы, p-лапласиан.

\section{§ 1. Введение}

Пусть $T=[0,1]$ - отрезок числовой прямой $\mathbb{R}, H$ - гильбертово пространство. Функция $\varphi: H \rightarrow(-\infty,+\infty]$ называется собственной, если она не равна тождественно $+\infty$, т.е. если ее эффективная область $\operatorname{dom} \varphi=\{x \in H ; \varphi(x)<+\infty\}$ не пуста. Множество всех функций $\varphi: H \rightarrow(-\infty,+\infty]$, которые являются собственными выпуклыми и полунепрерывными снизу, будем обозначать через $\Gamma_{0}(H)$. Для $\varphi \in \Gamma_{0}(H)$ через $\partial \varphi(x)$ будем обозначать ее субдифференциал в точке $x$ (см. [1]), а через $\operatorname{dom} \partial \varphi=\{x \in H ; \partial \varphi(x) \neq \varnothing\}$ - область определения $\partial \varphi$. Известно (см. [1]), что отображение $\partial \varphi: H \rightarrow H$, которое мы будем называть субдифференциалом функиии $\varphi$, является максимально монотонным оператором, $\operatorname{dom} \partial \varphi \subset \operatorname{dom} \varphi$ и $\overline{\operatorname{dom} \partial \varphi}=\overline{\operatorname{dom} \varphi}$, где черта означает замыкание в $H$.

Пусть $\varphi_{n}^{t} \in \Gamma_{0}(H), t \in T, n \geqslant 1$. При соответствующих предположениях относительно зависимости $\varphi_{n}^{t}, n \geqslant 1$, от $t$ мы можем определить функционалы

$$
\Phi_{n}(x)=\int_{T} \varphi_{n}^{t}(x(t)) d t, \quad x \in L^{2}(T, H) .
$$

Работа выполнена при финансовой поддержке Российского фонда фундаментальных исследований (грант № 06-01-00247) и Программы Президента РФ поддержки ведущих научных школ (грант № НШ-1676.2008.1).

(C) А. А. Толстоногов, 2009 
Нас будут интересовать вопросы сходимости по Моско (см. [2]) последовательности $\Phi_{n}, n \geqslant 1$, при $n \rightarrow \infty$.

Со сходимостью по Моско последовательности $\Phi_{n}, n \geqslant 1$, тесно связана задача о предельных переходах при $n \rightarrow \infty$ в эволюционных включениях в гильбертовом пространстве

$$
-\dot{x}_{n}(t) \in \partial \varphi_{n}^{t}\left(x_{n}(t)\right)+f_{n}(t), \quad n \geqslant 1, \quad f_{n} \in L^{2}(T, H) .
$$

Вопрос о том, что является пределом последовательности решений $x_{n}(\cdot), n \geqslant$ 1 , включения (1.2), возникает при изучении различных физических задач (см. [3]-[8] и др.).

В качестве примера мы рассматриваем следующую задачу. Пусть $\Omega \subset R^{N}-$ ограниченная область с гладкой границей; $K^{t} \subset \mathbb{R}, t \in T,-$ зависящее от времени выпуклое замкнутое множество, содержащее нуль; $I\left(K^{t}\right)$ - индикаторная функция множества $K^{t}$ и $\partial I\left(K^{t}\right)$ - субдифференциал функции $I\left(K^{t}\right)$.

Возникает вопрос: что является пределом последовательности $u(p)(\cdot), N<$ $p<+\infty$, решений включений

$-\dot{u}_{t}(p)(t, x) \in-\operatorname{div}\left(\|\nabla u(p)(t, x)\|^{p-2} \nabla u(p)(t, x)\right)+\partial I\left(K^{t}\right)(u(p)(t, x))+f(p)(t, x)$

при $p \rightarrow+\infty$, когда $f(p)$ слабо сходится в $L^{2}(T \times \Omega)$ к $f$ ?

В приложении мы даем ответ на этот вопрос. Сходимость функционалов (1.1) и предельные переходы во включениях (1.2) с субдифференциальными операторами, зависящими от времени, изучались в [6], [9].

Предельный переход во включениях

$$
-u_{t}(p)(t, x) \in-\operatorname{div}\left(\left(\frac{1}{\gamma(x, t)}\right)^{p}\|\nabla u(p)(t, x)\|^{p-2} \nabla u(p)(t, x)\right)+f(p)(t, x)
$$

при $p \rightarrow \infty$, когда $f(p)$ сходится в $L^{2}\left(T, L^{2}(\Omega)\right)$ к $f$, рассматривался в [9]. Здесь $\gamma(x, t)=\pi(x) \theta(t), \pi \in L^{\infty}(\Omega), \theta \in W^{1,2}(T), \gamma(x, t) \geqslant \delta>0$ для почти всех $x \in \Omega$ и всех $t \in T$. Наши результаты обобщают и дополняют известные результаты подобного типа, в том числе результаты работ [6], [9].

\section{§ 2. Основные обозначения, определения и предложения}

Пусть $H$ - гильбертово пространство с нормой $\|\cdot\|$ и со скалярным произведением $\langle\cdot, \cdot\rangle$. Для банахова пространства $X$ символ $\omega$ - $X$ означает, что пространство $X$ наделено слабой $\sigma\left(X, X^{*}\right)$ топологией (см. [10]), где $X^{*}$ - пространство, топологически сопряженное к $X$. Такое же обозначение мы используем и для подмножеств пространства $X$ с топологией, индуцированной топологией пространства $\omega$ - $X$. В остальных случаях пространство $X$ и его подмножества наделены сильной (нормированной) топологией.

Пусть $W^{1,2}(T, H)$ - пространство абсолютно непрерывных функций $x: T \rightarrow H$ с производной $\dot{x}(\cdot) \in L^{2}(T, H)$. Через $C(T, H)$ мы обозначаем пространство непрерывных функций $x: T \rightarrow H$ с топологией равномерной сходимости на $T$.

Многозначное отображение $F: H \rightarrow H$ называется полунепрерывным снизу nо Bъеторису в точке $x_{0} \in H$, если для любого открытого множества $U \subset H$, 
$F\left(x_{0}\right) \cap U \neq \varnothing$, существует окрестность $V\left(x_{0}\right)$ точки $x_{0}$ такая, что $F(x) \cap U \neq \varnothing$ для любого $x \in V\left(x_{0}\right)$.

Воспользовавшись утверждением 3.19 из [2], введем следующее

ОПРЕДЕЛЕНИЕ. Пусть $X$ - рефлексивное банахово пространство, $F_{n}: X \rightarrow$ $(-\infty,+\infty], n \geqslant 1,-$ последовательность функций. Последовательность $F_{n}$, $n \geqslant 1$, сходится к $F: X \rightarrow(-\infty,+\infty]$ в смысле Моско $\left(F_{n} \stackrel{M}{\longrightarrow} F\right)$, если:

1) для любого $x \in X$ существует последовательность $x_{n} \rightarrow x$ такая, что $F_{n}\left(x_{n}\right) \rightarrow F(x)$

2) для любого $x \in X$ и любой последовательности $x_{n} \in X, n \geqslant 1$, сходящейся к $x$ в пространстве $\omega-H$, имеет место неравенство

$$
F(x) \leqslant \varliminf_{n \rightarrow \infty} F_{n}\left(x_{n}\right)
$$

Отметим, что введенное в [2] определение сходимости по Моско (определение 3.17 ) отличается от нашего. Однако согласно утверждению 3.19 из [2] эти определения эквивалентны.

Пусть $\varphi \in \Gamma_{0}(H)$. Поскольку $\partial \varphi$ является максимально монотонным оператором (см. [1]), то для него будут определены операторы из $H$ в $H$ :

$$
J_{\lambda}=(I+\lambda \partial \varphi)^{-1}, \quad(\partial \varphi)_{\lambda}=\frac{1}{\lambda}\left(I-J_{\lambda}\right), \quad \lambda>0,
$$

где $I$ - тождественный оператор на $H$, которые называются резолъвентой и аппроксимацией Иосиды оператора $\partial \varphi$ соответственно. Эти операторы обладают следующими свойствами:

$$
\begin{gathered}
\left\|J_{\lambda}(x)-J_{\lambda}(y)\right\| \leqslant\|x-y\|, \quad\left\|(\partial \varphi)_{\lambda}(x)-(\partial \varphi)_{\lambda}(y)\right\| \leqslant \frac{1}{\lambda}\|x-y\|, \\
\lambda>0, \quad x, y \in H .
\end{gathered}
$$

Для каждого $\lambda>0$ регуляризачией Моро-Иосидь функции $\varphi \in \Gamma_{0}(H)$ называется функция

$$
\varphi_{\lambda}(x)=\inf \left\{\varphi(y)+\frac{1}{2 \lambda}\|y-x\|^{2}, y \in H\right\} .
$$

Следующие свойства функций $\varphi_{\lambda}$ и операторов $J_{\lambda},(\partial \varphi)_{\lambda}$ хорошо известны (см. [1], [9] и др). Поскольку эти свойства используются в наших доказательствах, приведем их.

УТВЕРЖДЕНИЕ. Пусть $\varphi \in \Gamma_{0}(H)$. Тогда:

(1) $\varphi_{\lambda}$ является конечной непрерывной и выпуклой функиией на $H$;

(2) $\varphi_{\lambda}$ является дифференцируемой по Фреше, и для ее производной $\partial \varphi_{\lambda}$ по Фреше имеет место равенство

$$
\partial \varphi_{\lambda}=(\partial \varphi)_{\lambda}, \quad \lambda>0
$$

(3) для любых $x \in H, \lambda>0$

$$
\varphi_{\lambda}(x) \leqslant \varphi(x), \quad \varphi_{\lambda}(x) \uparrow \varphi(x) \quad \text { npu } \lambda \downarrow 0 ;
$$


(4) если $x \in \operatorname{dom} \partial \varphi$, mo

$$
\left\|\partial \varphi_{\lambda}(x)\right\| \leqslant\left\|(\partial \varphi)^{\circ}(x)\right\|, \quad \partial \varphi_{\lambda}(x) \rightarrow(\partial \varphi)^{\circ}(x) \quad \text { npu } \quad \lambda \downarrow 0,
$$

где $(\partial \varphi)^{\circ}(x)$ - единственный элемент множества $\partial \varphi(x)$ такой, что

$$
\left\|(\partial \varphi)^{\circ}(x)\right\|=\min \{\|y\| ; y \in \partial \varphi(x)\} ;
$$

(5) для любъх $\lambda, \mu>0, x \in H$

$$
J_{\lambda}(x)=J_{\mu}\left(\frac{\mu}{\lambda} x+\left(1-\frac{\mu}{\lambda}\right) J_{\lambda}(x)\right) ;
$$

(6) для любъх $\lambda>0, x \in H$

$$
\varphi_{\lambda}(x)=\frac{1}{2 \lambda}\left\|x-J_{\lambda}(x)\right\|^{2}+\varphi\left(J_{\lambda}(x)\right) ;
$$

(7) для любъх $\lambda>0, x, y \in H$

$$
\varphi_{\lambda}(x) \leqslant \varphi_{\lambda}(y)+\left\|\partial \varphi_{\lambda}(x)\right\|\|x-y\| ;
$$

(8) если $\lambda_{n} \downarrow 0$ u $x_{n} \in H, n \geqslant 1, x_{n} \rightarrow x$, mo

$$
\varphi(x) \leqslant \lim _{n \rightarrow \infty} \varphi_{\lambda_{n}}\left(x_{n}\right) .
$$

Пусть $\Lambda$ - компактное метрическое пространство и $\varphi^{t}(p) \in \Gamma_{0}(H), t \in T$, $p \in \Lambda$. Рассмотрим включения

$$
\begin{gathered}
-\dot{x}(p)(t) \in \partial \varphi^{t}(p)(x(p)(t))+f(t), \\
f \in L^{2}(T, H), \quad x(p)(0)=y(p) \in \operatorname{dom} \varphi^{0}(p), \quad p \in \Lambda .
\end{gathered}
$$

При фиксированном $p \in \Lambda$ под решением включения (2.10) понимается функция $x(p) \in W^{1,2}(T, H), x(p)(0)=y(p)$, такая, что $x(p)(t) \in \operatorname{dom} \partial \varphi^{t}(p)$ почти всюду и почти всюду на $T$ имеет место включение $(2.10)$.

Введем следующее

ПреДПОЛОЖениЕ $H(\varphi)$. Семейство функиий $\varphi^{t}(p) \in \Gamma_{0}(H), t \in T, p \in \Lambda$, обладает следующими свойствами:

(1) $\varphi^{t}\left(p_{n}\right) \stackrel{M}{\longrightarrow} \varphi^{t}(p), t \in T$, для любого $p \in \Lambda$ и любой последователъности $p_{n} \in \Lambda, n \geqslant 1, p_{n} \rightarrow p$

(2) существует $c_{0}>0$ такое, что

$$
\left\{x \in H ; \varphi^{0}(p)(x)<c_{0}\right\} \neq \varnothing \quad \forall p \in \Lambda ;
$$

(3) для любых $r>0, p \in \Lambda$ существуют функиии $a_{r}(p) \in W^{1,2}(T), b_{r}(p) \in$ $W^{1,1}(T)$ такие, что для каждых $s, t \in T, s \leqslant t, x \in \operatorname{dom} \varphi^{s}(p) c\|x\| \leqslant r$ найдется $x_{1} \in \operatorname{dom} \varphi^{t}(p)$, удовлетворяющее неравенствам

$$
\begin{gathered}
\left\|x_{1}-x\right\| \leqslant\left|a_{r}(p)(t)-a_{r}(p)(s)\right|\left(1+\left|\varphi^{s}(p)(x)\right|^{1 / 2}\right), \\
\varphi^{t}(p)\left(x_{1}\right)-\varphi^{s}(p)(x) \leqslant\left|b_{r}(p)(t)-b_{r}(p)(s)\right|\left(1+\left|\varphi^{s}(p)(x)\right|\right) ;
\end{gathered}
$$


(4) для каждого $r>0$ множества

$$
\left\{\dot{a}_{r}(p), p \in \Lambda\right\}, \quad\left\{\dot{b}_{r}(p), p \in \Lambda\right\}
$$

относительно компактны в $\omega-L^{2}(T)$ и $\omega-L^{1}(T)$ соответственно.

Всюду в дальнейшем мы предполагаем, что предположения $H(\varphi)$ имеют место.

\section{§ 3. Вспомогательные результаты}

Рассмотрим многозначное отображение $F(c): \Lambda \rightarrow H$, определенное по правилу

$$
F(c)(p)=\left\{x \in H ; \varphi^{0}(p)(x) \leqslant c\right\}, \quad c \geqslant c_{0}, \quad p \in \Lambda,
$$

где $c_{0}>0$ - константа, фигурирующая в неравенстве (2.11).

Лемма 3.1. Для любого $c \geqslant c_{0}$ многозначное отображение $F(c): \Lambda \rightarrow H$ является полунепрерывным снизу по Въеторису с замкнутыми выпуклыми значениями.

ДокАЗАтельство. Рассмотрим случай $c=c_{0}$, поскольку при $c>c_{0}$ доказательство проводится аналогично. Пусть

$$
U(p)=\left\{x \in H ; \varphi^{0}(p)(x)<c_{0}\right\} .
$$

Из (2.11) следует, что значениями отображения $p \rightarrow U(p)$ являются непустые выпуклые множества пространства $H$. Пусть $p \in \Lambda, y \in U(p), p_{n} \geqslant 1$ произвольны и $p_{n} \rightarrow p, n \rightarrow \infty$.

Так как $\varphi^{0}\left(p_{n}\right) \stackrel{M}{\longrightarrow} \varphi^{0}(p)$, то существует последовательность $y_{n} \rightarrow y, n \geqslant 1$, такая, что $\varphi^{0}\left(p_{n}\right)\left(y_{n}\right) \rightarrow \varphi^{0}(p)(y)$. Тогда согласно (3.2) при достаточно больших $n$ будет иметь место неравенство $\varphi\left(p_{n}\right)\left(y_{n}\right)<c_{0}$. Последнее означает, что $y_{n} \in U\left(p_{n}\right)$ при достаточно больших $n$. Поэтому отображение $p \rightarrow U(p)$ является полунепрерывным снизу по Вьеторису.

Пусть $y \in F\left(c_{0}\right)(p)$ и $x \in U(p)$. Рассмотрим последовательность $x_{n}=$ $\lambda_{n} y+\left(1-\lambda_{n}\right) x, \lambda_{n} \rightarrow 1,0<\lambda_{n}<1$. Из выпуклости функции $\varphi^{0}(p)$ следует, что $\varphi^{0}(p)\left(x_{n}\right)<c_{0}$. Поэтому $x_{n} \in U(p), n \geqslant 1$, и $x_{n} \rightarrow y \in F\left(c_{0}\right)(p)$. Так как $F\left(c_{0}\right)(p)$ является выпуклым замкнутым множеством, то $F\left(c_{0}\right)(p)=\overline{U(p)}$, $p \in \Lambda$, где черта сверху означает замыкание в $H$. Поскольку многозначное отображение $p \rightarrow U(p)$ полунепрерывно снизу по Вьеторису, то и многозначное отображение $p \rightarrow F\left(c_{0}\right)(p)=\overline{U(p)}$ является полунепрерывным снизу по Вьеторису с замкнутыми выпуклыми значениями. Лемма доказана.

СлеДСТВИЕ 3.1. Для любых $p_{0} \in \Lambda, c \geqslant c_{0}, y_{0} \in H, \varphi^{0}\left(p_{0}\right)\left(y_{0}\right) \leqslant c$ cyществует непрерьвная функиия $y^{0}: \Lambda \rightarrow H$ такая, что

$$
y^{0}\left(p_{0}\right)=y_{0}, \quad \varphi^{0}(p)\left(y^{0}(p)\right) \leqslant c, \quad p \in \Lambda .
$$


Поскольку $\varphi^{0}\left(p_{0}\right)\left(y_{0}\right) \leqslant c$, то $y_{0} \in F(c)\left(p_{0}\right)$, где $F(c)(p)$ - многозначное отображение, определенное по правилу (3.1). Так как отображение $p \rightarrow F(c)(p)$ полунепрерывно снизу по Вьеторису с замкнутыми выпуклыми значениями, то согласно теореме Майкла (см. [11]) о непрерывных селекторах существует непрерывная функция $y^{0}: \Lambda \rightarrow H$ такая, что $y^{0}\left(p_{0}\right)=y_{0}$ и $y^{0}(p) \in F(c)(p)$, $p \in \Lambda$. Последнее означает, что имеют место соотношения (3.3).

Лемма 3.2. Существует $\alpha^{0}>0$ maкое, что

$$
\varphi^{0}(p)(x)+\alpha^{0}\|x\|+\alpha^{0} \geqslant 0, \quad x \in H, \quad p \in \Lambda .
$$

ДокАзАТЕЛЬство. Проведем его от противного. Предположим, что неравенство (3.4) не имеет места. Тогда существуют последовательности $p_{n} \in \Lambda$, $x_{n} \in H, n \geqslant 1$, такие, что

$$
\varphi^{0}\left(p_{n}\right)\left(x_{n}\right)+n\left(\left\|x_{n}\right\|+1\right)<0 .
$$

Возможны два случая.

Случай 1. Последовательность $x_{n}, n \geqslant 1$, ограничена. Не нарушая общности, будем считать, что $p_{n} \rightarrow p$ и $x_{n} \rightarrow x$ в $\omega-H$. Так как $\varphi^{0}\left(p_{n}\right) \stackrel{M}{\longrightarrow} \varphi^{0}(p)$, то из (3.5) следует

$$
\varphi^{0}(p)(x) \leqslant \underline{\lim _{n \rightarrow \infty}} \varphi^{0}\left(p_{n}\right)\left(x_{n}\right) \leqslant-\infty .
$$

Поскольку $\varphi^{0}(p)(x) \in(-\infty,+\infty]$, то последнее неравенство приводит нас к противоречию.

Случай 2. Последовательность $x_{n}, n \geqslant 1$, не ограничена. Тогда мы можем считать, что $p_{n} \rightarrow p$ и $\left\|x_{n}\right\| \rightarrow+\infty$. Пусть $y^{0}: \Lambda \rightarrow H-$ непрерывная функция, удовлетворяющая неравенству (3.3) при $c=c_{0}$. Положим

$$
\beta_{n}=\left(\sqrt{n}\left\|x_{n}-y^{0}\left(p_{n}\right)\right\|\right)^{-1}, \quad z_{n}=\beta_{n} x_{n}+\left(1-\beta_{n}\right) y^{0}\left(p_{n}\right) .
$$

Поскольку $\left\|x_{n}\right\| \rightarrow+\infty$ и $y^{0}\left(p_{n}\right) \rightarrow y^{0}(p), n \rightarrow \infty$, то $\beta_{n} \in(0,1)$ при достаточно больших $n$. Из равенства

$$
\left\|z_{n}-y^{0}\left(p_{n}\right)\right\|=\beta_{n}\left\|x_{n}-y^{0}\left(p_{n}\right)\right\|=\frac{1}{\sqrt{n}}
$$

следует, что $z_{n} \rightarrow y^{0}(p)$ при $n \rightarrow+\infty$. Воспользовавшись выпуклостью функций $\varphi^{0}\left(p_{n}\right), n \geqslant 1$, и неравенством $(3.5)$, получаем, что при достаточно больших $n$

$$
\begin{aligned}
\varphi^{0}\left(p_{n}\right)\left(z_{n}\right) & \leqslant \beta_{n} \varphi^{0}\left(p_{n}\right)\left(x_{n}\right)+\left(1-\beta_{n}\right) \varphi^{0}\left(p_{n}\right)\left(y^{0}\left(p_{n}\right)\right) \\
& \leqslant-\sqrt{n} \frac{\left\|x_{n}\right\|+1}{\left\|x_{n}-y^{0}\left(p_{n}\right)\right\|}+\left(1-\beta_{n}\right) \varphi^{0}\left(p_{n}\right)\left(y^{0}\left(p_{n}\right)\right) .
\end{aligned}
$$

Из этого неравенства, сходимости $\varphi^{0}\left(p_{n}\right) \stackrel{M}{\longrightarrow} \varphi^{0}(p)$ и неравенства в (3.3) вытекает, что

$$
\varphi^{0}(p)\left(y^{0}(p)\right) \leqslant \varliminf_{n \rightarrow \infty} \varphi^{0}\left(p_{n}\right)\left(z_{n}\right) \leqslant-\infty .
$$

Последнее неравенство приводит нас к противоречию. Лемма доказана. 
Лемма 3.3. Существуют константа $C$ инепрерьвнъе функции $y^{t}: \Lambda \rightarrow H$, $t \in T$, maкue, чmo

$$
\left\|y^{t}(p)\right\|<C, \quad \varphi^{t}(p)\left(y^{t}(p)\right)<C, \quad p \in \Lambda, \quad t \in T .
$$

ДокАЗАТЕЛЬство. Из лемм 3.1, 3.2 следует, что существуют $c_{1}>c_{0}$ и непрерывная функция $y^{0}: \Lambda \rightarrow H$ такие, что

$$
\left\|y^{0}(p)\right\| \leqslant c_{1}, \quad\left|\varphi^{0}(p)\left(y^{0}(p)\right)\right| \leqslant c_{1}, \quad p \in \Lambda .
$$

Воспользовавшись $(2.12),(2.13)$ и $(3.7)$, мы получаем, что существует $c_{2}>0$ такое, что для любых $t \in T, p \in \Lambda$ найдется $z^{t}(p) \in H$ такое, что

$$
\left\|z^{t}(p)\right\|<c_{2}, \quad \varphi^{t}(p)\left(z^{t}(p)\right)<c_{2} .
$$

Пусть $U^{t}(p)=\left\{x \in H: \varphi^{t}(p)(x)<c_{2}\right\}$ и $B_{c_{2}}=\left\{x \in H:\|x\|<c_{2}\right\}$. Как и при доказательстве леммы 3.1, мы получаем, что отображение $p \rightarrow U^{t}(p)$, $t \in T$, является полунепрерывным снизу по Вьеторису с непустыми выпуклыми значениями. Так как $z^{t}(p) \in U^{t}(p) \cap B_{c_{2}}, p \in P, t \in T$, то отображение $p \rightarrow$ $U^{t}(p) \cap B_{c_{2}}, t \in T$, является полунепрерывным снизу по Вьеторису с непустыми выпуклыми значениями. Таковым является и многозначное отображение $p \rightarrow$ $\overline{U^{t}(p) \cap B_{c_{2}}}$, где черта означает замыкание в $H$. Согласно теореме Майкла (см. [11]) для каждого $t \in T$ существует непрерывный селектор $y^{t}: \Lambda \rightarrow H$ отображения $p \rightarrow \overline{U^{t}(p) \cap B_{c_{2}}}$. Ясно, что функции $y^{t}, t \in T$, удовлетворяют неравенствам (3.6) при $C>c_{2}$. Лемма доказана.

Лемма 3.4. Для любого $t \in T$ существует $\alpha^{t}>0$ такое, что для любых $x \in H$ u $p \in \Lambda$ имеет место неравенство

$$
\varphi^{t}(p)(x)+\alpha^{t}\|x\|+\alpha^{t} \geqslant 0 .
$$

Доказательство леммы дословно повторяет доказательство леммы 3.2 с использованием неравенств (3.6).

Teоpema 3.1. Cуществует $\alpha>0$ maкoе, чmo

$$
\varphi^{t}(p)(x)+\alpha\|x\|+\alpha \geqslant 0, \quad t \in T, \quad p \in \Lambda, \quad x \in H .
$$

ДокАЗАТЕЛЬСтво. Положим $r=C+1$, где $C$ - константа из неравенств (3.6). Воспользовавшись свойством (4) предположения $H(\varphi)$ и теоремой Данфорда-Петтиса (см. [12; теорема 4.21.2]), выберем разбиение $0=s_{0}<s_{1}<$ $\cdots<s_{n}=1$ отрезка $T=[0,1]$ так, чтобы

$$
\begin{gathered}
\left|a_{r}(p)\left(s_{i}\right)-a_{r}(p)(t)\right| \leqslant \frac{1}{2}, \quad\left|b_{r}(p)\left(s_{i}\right)-b_{r}(p)(t)\right| \leqslant \frac{1}{2}, \\
i=1, \ldots, n, \quad t \in\left[s_{i-1}, s_{i}\right], \quad p \in \Lambda .
\end{gathered}
$$

Пусть $p \in \Lambda, t \in[0,1)$ произвольны и фиксированы. Тогда $t \in\left[s_{i-1}, s_{i}\right)$ при некотором $1 \leqslant i \leqslant n$. Согласно лемме 3.3 существует $x \in \operatorname{dom} \varphi^{t}(p)$ с $\|x\| \leqslant r$. 
Используя неравенства $(2.12),(2.13)$ и (3.10), мы получим, что для каждого $x \in \operatorname{dom} \varphi^{t}(p),\|x\| \leqslant r$, найдется $\widetilde{x} \in \operatorname{dom} \varphi^{s_{i}}(p)$ такое, что

$$
\begin{gathered}
\|\widetilde{x}-x\| \leqslant \frac{1}{2}\left(1+\left|\varphi^{t}(p)(x)\right|^{1 / 2}\right), \\
\varphi^{t}(p)(x) \geqslant \varphi^{s_{i}}(p)(\widetilde{x})-\frac{1}{2}\left(1+\left|\varphi^{t}(p)(x)\right|\right) .
\end{gathered}
$$

Согласно лемме 3.4 существует $m>0$ такое, что

$$
\varphi^{s_{i}}(p)(x) \geqslant-m\|x\|-m, \quad x \in H, \quad p \in \Lambda, \quad i=0, \ldots, n .
$$

Из неравенства (3.11) и неравенства Коши следует, что

$$
\|x-\widetilde{x}\| \leqslant \frac{1}{2}+\frac{m}{4}+\frac{1}{4 m}\left|\varphi^{t}(p)(x)\right| .
$$

Воспользовавшись (3.12)-(3.14), мы получаем

$$
\varphi^{t}(p)(x)+\frac{3}{4}\left|\varphi^{t}(p)(x)\right| \geqslant-m\|x\|-\frac{3}{2} m-\frac{m^{2}}{4}-\frac{1}{2}, \quad p \in \Lambda, \quad t \in[0,1) .
$$

Из последнего неравенства и неравенства (3.8) при $t=1$ вытекает, что существует $\delta>0$ такое, что

$$
\varphi^{t}(p)(x) \geqslant-\delta\|x\|-\delta, \quad t \in T, \quad p \in \Lambda, \quad x \in \operatorname{dom} \varphi^{t}(p), \quad\|x\| \leqslant r .
$$

Пусть $t \in T, p \in \Lambda$ произвольны и фиксированы. Возьмем $x \in \operatorname{dom} \varphi^{t}(p)$ с $\|x\|>r=C+1$. Согласно лемме 3.3 существует $z^{t} \in \operatorname{dom} \varphi^{t}(p)$ с $\left\|z^{t}\right\|<C$ и $\varphi^{t}(p)\left(z^{t}\right)<C$. Положим

$$
x^{t}=\lambda x+(1-\lambda) z^{t}, \quad \lambda=\frac{1}{\left\|z^{t}-x\right\|} .
$$

Поскольку $\|x\|>C+1$ и $\left\|z^{t}\right\|<C$, то $0<\lambda<1$. Поэтому $x^{t} \in \operatorname{dom} \varphi^{t}(p)$ и $\left\|x^{t}\right\|<r$. Тогда в соответствии с (3.15)

$$
\lambda \varphi^{t}(p)(x)+(1-\lambda) \varphi^{t}(p)\left(z^{t}\right)+\delta\left\|x^{t}\right\|+\delta \geqslant \varphi^{t}(p)\left(x^{t}\right)+\delta\left\|x^{t}\right\|+\delta \geqslant 0 .
$$

Из этого неравенства вытекает, что

$$
\varphi^{t}(p)(x)+\frac{1}{\lambda}\left\{(1-\lambda) \varphi^{t}(p)\left(z^{t}\right)+\delta\left\|x^{t}\right\|+\delta\right\} \geqslant 0 .
$$

Поскольку $\lambda^{-1}=\left\|z^{t}-x\right\| \leqslant\|x\|+C$ и

$$
(1-\lambda) \varphi^{t}(p)\left(z^{t}\right)+\delta\left\|x^{t}\right\|+\delta \leqslant(1-\lambda) C+\delta(C+1)+\delta,
$$

Tо

$$
\varphi^{t}(p)(x)+(\|x\|+C)\{C+\delta(1+C)+\delta\} \geqslant 0 .
$$

Теперь из (3.15), (3.16) вытекает, что существует $\alpha>0$ такое, что неравенство (3.9) имеет место для любых $p \in \Lambda, t \in T$ и $x \in \operatorname{dom} \varphi^{t}(p)$. Если $x \notin$ $\operatorname{dom} \varphi^{t}(p)$, то неравенство (3.9) выполняется автоматически. Теорема доказана. 
СледСТВИЕ 3.2. Существуют число $R>0$ u непрерывные функции $y^{t}$ : $\Lambda \rightarrow H, t \in T$, удовлетворяющие неравенствам

$$
\left\|y^{t}(p)\right\|<R, \quad\left|\varphi^{t}(p)\left(y^{t}(p)\right)\right|<R, \quad p \in \Lambda, \quad t \in T .
$$

Следствие вытекает из леммы 3.3 и теоремы 3.1 .

Лемма 3.5. Существует константа $K>0$ такая, что

$$
\begin{gathered}
\left\|J_{\lambda}^{t}(p)(x)\right\| \leqslant K+\|x\|, \\
\left\|\partial \varphi_{\lambda}^{t}(p)(x)\right\| \leqslant \frac{1}{\lambda}(K+2\|x\|), \quad x \in H, \quad t \in T, \quad p \in \Lambda, \quad \lambda \in(0,1] .
\end{gathered}
$$

ДокАЗАТЕльСтво. Воспользовавшись (2.4), (2.7), (3.9) и (3.17), мы получим при $\lambda=1$

$$
\begin{aligned}
R \geqslant \varphi_{1}^{t}(p)\left(y^{t}(p)\right) & =\varphi^{t}(p)\left(J_{1}^{t}(p)\left(y^{t}(p)\right)\right)+\frac{1}{2}\left\|y^{t}(p)-J_{1}^{t}(p)\left(y^{t}(p)\right)\right\|^{2} \\
& \geqslant-\alpha\left\|J_{1}^{t}(p)\left(y^{t}(p)\right)\right\|-\alpha+\frac{1}{2}\left\|y^{t}(p)-J_{1}^{t}(p)\left(y^{t}(p)\right)\right\|^{2} .
\end{aligned}
$$

Из этого неравенства и (3.17) вытекает, что

$$
\left\|J_{1}^{t}(p)\left(y^{t}(p)\right)\right\| \leqslant d, \quad p \in \Lambda, \quad t \in T,
$$

при некотором $d>0$.

Принимая во внимание первое неравенство (2.2), (2.6) и (3.20), мы приходим к неравенству

$$
\begin{aligned}
\left\|J_{\lambda}^{t}(p)\left(y^{t}(p)\right)\right\| & \leqslant\left\|J_{1}^{t}(p)\left(y^{t}(p)\right)\right\|+\left\|J_{\lambda}^{t}(p)\left(y^{t}(p)\right)-J_{1}^{t}(p)\left(y^{t}(p)\right)\right\| \\
& \leqslant\left\|J_{1}^{t}(p)\left(y^{t}(p)\right)\right\|+(1-\lambda)\left\|y^{t}(p)-J_{1}^{t}(p)\left(y^{t}(p)\right)\right\| \\
& \leqslant 2 d+\left\|y^{t}(p)\right\|, \quad p \in \Lambda, \quad \lambda \in(0,1] .
\end{aligned}
$$

Теперь из (2.2), (3.21) вытекает

$$
\left\|J_{\lambda}^{t}(p)(x)\right\| \leqslant\left\|J_{\lambda}^{t}(p)\left(y^{t}(p)\right)\right\|+\left\|x-y^{t}(p)\right\| \leqslant 2 d+2 R+\|x\|, \quad \lambda \in(0,1] .
$$

Полагая $K=2 d+2 R$ в последнем неравенстве, мы приходим к неравенству (3.18). Неравенство (3.19) вытекает из второго неравенства (2.1) и (3.18). Лемма доказана.

\section{§ 4. Априорные оценки}

Пусть $r=R+1, M=R+\alpha r+\alpha+1$, где $R$ - константа из неравенства (3.17), $0 \leqslant t_{0}<1$ произвольно и $y^{t_{0}}: \Lambda \rightarrow H-$ непрерывная функция, удовлетворяющая неравенствам (3.17). Воспользовавшись свойством (4) предположения $H(\varphi)$, мы получим, что существует $t_{1}>t_{0}$ такое, что

$$
\left\{1+M \exp \left(\int_{0}^{1}\left|\dot{b}_{r}(p)(\tau)\right| d \tau\right)\right\} \int_{t_{0}}^{t_{1}}\left|\dot{a}_{r}(p)(\tau)\right| d \tau \leqslant 1, \quad p \in \Lambda .
$$


Лемма 4.1. Существует функиия $h(p) \in W^{1,2}\left(\left[t_{0}, t_{1}\right], H\right), p \in \Lambda$, удовлетворяющая соотношениям

$$
\begin{gathered}
h(p)\left(t_{0}\right)=y^{t_{0}}(p), \quad p \in \Lambda \\
\|h(p)(t)\| \leqslant r, \quad t \in\left[t_{0}, t_{1}\right], \quad p \in \Lambda ; \\
\left|\varphi^{t}(p)(h(p)(t))\right| \leqslant M+M \exp \left(\int_{0}^{1}\left|\dot{b}_{r}(p)(\tau)\right| d \tau\right) \int_{t_{0}}^{t}\left|\dot{b}_{r}(p)(\tau)\right| d \tau, \\
p \in \Lambda, \quad t \in\left[t_{0}, t_{1}\right] ; \\
\|\dot{h}(p)(t)\| \leqslant\left\{\begin{array}{c}
\left.1+M \exp \left(\int_{0}^{1}\left|\dot{b}_{r}(p)(\tau)\right| d \tau\right)\right\}\left|\dot{a}_{r}(t)\right|, \\
p \in \Lambda, \quad \text { п.в. на }\left[t_{0}, t_{1}\right] .
\end{array}\right.
\end{gathered}
$$

Подобная лемма была доказана в [9] для случая, когда функции $\varphi^{t}(p)$ не зависят от $p$, т.е. $\varphi^{t}(p)=\varphi^{t}, t \in T, p \in \Lambda$. Доказательство нашей леммы дословно повторяет доказательство леммы 1.5.3 из [9] с использованием свойства (4) предположения $H(\varphi)$ и теоремы 3.1.

Лемма 4.2. Существуют разбиение $0=t_{0}<t_{1}<\cdots<t_{N}=1$ отрезка $T=[0,1]$ и функция $h(p): T \rightarrow H, p \in \Lambda$, такие, что: $h(p), p \in \Lambda$, непрерывна на отрезке $\left[t_{0}, t_{1}\right]$ и на каждом полуинтервале $\left(t_{k-1}, t_{k}\right], k=2, \ldots, N$, разбиения; бункция $h(p), p \in \Lambda$, абсолютно непрерывна на каждом интервале $\left(t_{k-1}, t_{k}\right), k=1, \ldots, N ;$ бункция $p \rightarrow h(p)\left(t_{k-1}\right), k=1, \ldots, N$, непрерывна и справедливы неравенства

$$
\begin{gathered}
\|h(p)(t)\| \leqslant r, \quad t \in T, \quad p \in \Lambda \\
\left|\varphi^{t}(p)(h(p)(t))\right| \leqslant M+M \exp \left(\int_{0}^{1}\left|\dot{b}_{r}(p)(\tau)\right| d \tau\right) \int_{0}^{t}\left|\dot{b}_{r}(p)(\tau)\right| d \tau, \\
t \in T, \quad p \in \Lambda ; \\
\|\dot{h}(p)(t)\| \leqslant\left\{1+M \exp \left(\int_{0}^{1}\left|\dot{b}_{r}(p)(\tau)\right| d \tau\right)\right\}\left|\dot{a}_{r}(p)(t)\right|, \\
p \in \Lambda, \text { п.в. на T. }
\end{gathered}
$$

ДокАЗАТЕЛЬСтво. Возьмем $r=R+1, M=R+\alpha r+\alpha+1$ и выберем разбиение $0=t_{0}<t_{1}<\cdots<t_{N}=1$ отрезка $T=[0,1]$ так, чтобы

$$
\begin{gathered}
\left\{1+M \exp \left(\int_{0}^{1}\left|\dot{b}_{r}(p)(\tau)\right| d \tau\right)\right\} \int_{t_{k-1}}^{t_{k}}\left|\dot{a}_{r}(p)(\tau)\right| d \tau \leqslant 1, \\
k=1, \ldots, N, \quad p \in \Lambda .
\end{gathered}
$$

Существование такого разбиения следует из свойства (4) предположения $H(\varphi)$. Пусть $y^{t_{k}}: \Lambda \rightarrow H, k=0, \ldots, N-1,-$ непрерывная функция, удовлетворяющая неравенствам (3.17). Тогда согласно лемме 4.1 существуют функции $h_{k}(p) \in W^{1,2}\left(\left[t_{k-1}, t_{k}\right], H\right), k=1, \ldots, N, p \in \Lambda$, такие, что $h_{k}(p)\left(t_{k-1}\right)=y^{t_{k-1}}(p)$, $p \in \Lambda, k=1, \ldots, N$, и на каждом из отрезков $\left[t_{k-1}, t_{k}\right]$ функции $h_{k}(p)$ удовлетворяют неравенствам, аналогичным неравенствам (4.2)-(4.4). Теперь построение функции $h(p)$ с требуемыми свойствами очевидно. Лемма доказана. 
Из предположений $H(\varphi)$, теоремы 1.5.1 и леммы 1.2.2 из [9] вытекает, что для любой функции $x \in L^{2}(T, H)$ функции $t \rightarrow \varphi^{t}(p)(x(t)), t \rightarrow \varphi_{\lambda}^{t}(p)(x(t))$, $t \rightarrow \partial \varphi_{\lambda}^{t}(p)(x(t)), t \rightarrow J_{\lambda}^{t}(p)(x(t)), p \in \Lambda, \lambda \in(0,1]$, измеримы.

Пусть $y^{0}: \Lambda \rightarrow H-$ непрерывная функция, удовлетворяющая неравенствам (3.17).

Рассмотрим включение

$$
\begin{gathered}
-\dot{x}(p)(t) \in \partial \varphi^{t}(p)(x(p)(t))+f(t), \\
x(p)(0)=y^{0}(p) \in \operatorname{dom} \varphi^{0}(p), \quad p \in \Lambda, \quad f \in L^{2}(T, H),
\end{gathered}
$$

и уравнение

$$
\begin{gathered}
-\dot{x}_{\lambda}(p)(t)=\partial \varphi_{\lambda}^{t}(p)\left(x_{\lambda}(p)(t)\right)+f(t), \\
x_{\lambda}(p)(0)=y^{0}(p), \quad p \in \Lambda, \quad \lambda \in(0,1] .
\end{gathered}
$$

При фиксированном $p \in \Lambda$ под решением уравнения (4.9) понимается функция $x_{\lambda}(p) \in W^{1,2}(T, H), x_{\lambda}(p)(0)=y^{0}(p)$, почти всюду на $T$ удовлетворяющая (4.9).

Хорошо известно (см. [9] и др.), что при выполнении предположений $H(\varphi)$ для любых $p \in \Lambda, f \in L^{2}(T, H), \lambda \in(0,1]$ включение (4.8) и уравнение (4.9) имеют единственные решения $x(p)$ и $x_{\lambda}(p)$.

В дальнейшем, чтобы подчеркнуть зависимость решений включения (4.8) и уравнения (4.9) от $p$ и $f$, мы их будем обозначать через $x(p, f)$ и $x_{\lambda}(p, f)$.

Пусть

$$
\begin{gathered}
S_{L}=\left\{f \in L^{2}(T, H) ;\|f\|_{L^{2}(T, H)} \leqslant L\right\}, \quad L>0, \\
\mathscr{R}_{L}=\left\{x(p, f) ; p \in \Lambda, f \in S_{L}\right\}, \\
\mathscr{R}_{L}^{*}=\left\{x_{\lambda}(p, f) ; p \in \Lambda, f \in S_{L}, \lambda \in(0,1]\right\} .
\end{gathered}
$$

Теорема 4.1. Существует константа $N>0$, зависящая только от $L$, такая, что

$$
\begin{gathered}
\left\|x_{\lambda}(p, f)(t)\right\| \leqslant N, \\
\left\|\dot{x}_{\lambda}(p, f)\right\|_{L^{2}(T, H)} \leqslant N, \\
\left|\varphi_{\lambda}^{t}(p)\left(x_{\mu}(p, f)(t)\right)\right| \leqslant N, \quad t \in T, \quad \lambda, \mu \in(0,1], \quad p \in \Lambda, \quad f \in S_{L} .
\end{gathered}
$$

ДоказАтельство. Пусть $x_{\lambda}(p, f) \in \mathscr{R}_{L}^{*}, 0=t_{0}<t_{1}<\cdots<t_{N-1}=1-$ разбиение отрезка $T$ и соответствующая этому разбиению функция $h(p): T \rightarrow H$ со свойствами, установленными в лемме 4.2. Тогда для почти каждого $s \in$ $\left[t_{k-1}, t_{k}\right], k=1, \ldots, N$, используя понятие субдифференциала выпуклой функции, мы получим

$$
\begin{gathered}
\frac{d}{d s}\left\|x_{\lambda}(p, f)(s)-h(p)(s)\right\|^{2}=2\left\langle\dot{x}_{\lambda}(p, f)(s)-\dot{h}(p)(s), x_{\lambda}(p, f)(s)-h(p)(s)\right\rangle \\
=-2\left\langle\partial \varphi_{\lambda}^{s}(p)\left(x_{\lambda}(p, f)(s)\right), x_{\lambda}(p, f)(s)-h(p)(s)\right\rangle \\
\quad-2\left\langle f(s)+\dot{h}(p)(s), x_{\lambda}(p, f)(s)-h(p)(s)\right\rangle \\
\leqslant 2 \varphi_{\lambda}^{s}(h(p)(s))-2 \varphi_{\lambda}^{s}(p)\left(x_{\lambda}(p, f)(s)\right) \\
\quad+2(\|f(s)\|+\|\dot{h}(p)(s)\|)\left\|x_{\lambda}(p, f)(s)-h(p)(s)\right\| .
\end{gathered}
$$


Из (2.7), (3.9), (3.18) вытекает, что

$$
\begin{aligned}
\varphi_{\lambda}^{s}(p) & \left(x_{\lambda}(p, f)(s)\right) \geqslant \varphi^{s}\left(J_{\lambda}^{s}(p)\left(x_{\lambda}(p, f)(s)\right)\right) \\
& \geqslant-\alpha\left\|J_{\lambda}^{s}(p)\left(x_{\lambda}(p, f)(s)\right)\right\|-\alpha \geqslant-\alpha\left(K+\left\|x_{\lambda}(p, f)(s)\right\|\right)-\alpha \\
& \geqslant-\alpha\left\|x_{\lambda}(p, f)(s)-h(p)(s)\right\|-\alpha\|h(p)(s)\|-\alpha K-\alpha \\
& \geqslant-\frac{1}{2}\left\|x_{\lambda}(p, f)(s)-h(p)(s)\right\|^{2}-\alpha\left(\frac{\alpha}{2}+\|h(p)(s)\|+K+1\right) .
\end{aligned}
$$

Воспользовавшись (4.16), (4.17), мы получим

$$
\frac{d}{d s}\left\|x_{\lambda}(p, f)(s)-h(p)(s)\right\|^{2} \leqslant 2\left\|x_{\lambda}(p, f)(s)-h(p)(s)\right\|^{2}+g(p)(s) \text { п.в. на } T,
$$

где

$$
\begin{gathered}
g(p)(s)=2\left|\varphi^{s}(p)(h(p)(s))\right|+(\|f(s)\|+\|\dot{h}(p)(s)\|)^{2} \\
+2 \alpha\|h(p)(s)\|+2 \alpha K+2 \alpha+\alpha^{2} .
\end{gathered}
$$

Теперь, дословно повторяя доказательство леммы 1.3.1 из [9] с использованием неравенств (3.17), (4.5)-(4.7), (4.10), (4.18), мы получим, что существует $N_{1}>0$, при котором имеет место неравенство

$$
\left\|x_{\lambda}(p, f)(t)\right\| \leqslant N_{1}, \quad t \in T, \quad \lambda \in(0,1], \quad p \in \Lambda, \quad f \in S_{L} .
$$

Так как $x_{\mu}(p, f) \in W^{1,2}(T, H), 0<\mu \leqslant 1$, то из леммы 2.2 в [13] вытекает, что

$$
\begin{gathered}
\varphi_{\lambda}^{t}(p)\left(x_{\mu}(p, f)(t)\right)+\int_{0}^{t}\left\langle\dot{x}_{\mu}(p, f)(s), \dot{x}_{\mu}(p, f)(s)+f(s)\right\rangle d s \\
\leqslant \varphi_{\lambda}^{0}(p)\left(y^{0}(p)\right)+\int_{0}^{t}\left|\dot{a}_{r}(p)(s)\right|\left\|\dot{x}_{\mu}(p, f)(s)+f(s)\right\| \\
\times\left(1+\left|\varphi_{\lambda}^{s}(p)\left(x_{\mu}(p, f)(s)\right)\right|^{1 / 2}\right) d s \\
+\int_{0}^{t}\left|\dot{b}_{r}(p)(s)\right|\left(1+\left|\varphi_{\lambda}^{s}(p)\left(x_{\mu}(p, f)(s)\right)\right|\right) d s,
\end{gathered}
$$

где

$$
r \geqslant \sup \left\{\left\|J_{\lambda}^{s}(p)\left(x_{\mu}(p, f)(s)\right)\right\| ; s \in T, 0<\lambda \leqslant 1\right\} .
$$

Воспользовавшись неравенством Коши, примененному к первому интегральному члену в правой части неравенства (4.20), и (3.17), (3.18), (4.19), (4.21), мы получим, что

$$
\begin{aligned}
\varphi_{\lambda}^{t}(p)\left(x_{\mu}(p, f)(t)\right)+\frac{1}{2} \int_{0}^{t}\left\|\dot{x}_{\mu}(p, f)(s)\right\|^{2} d s & \\
\leqslant R & +\frac{1}{2} \int_{0}^{t}\|f(s)\|^{2} d s+\int_{0}^{t}\left(\left|\dot{a}_{r}(p)(s)\right|^{2}+\left|\dot{b}_{r}(p)(s)\right|\right) \\
& \times\left(1+\left|\varphi_{\lambda}^{s}(p)\left(x_{\mu}(p, f)(s)\right)\right|\right) d s, \quad 0<\mu \leqslant 1,
\end{aligned}
$$


где

$$
r \geqslant K+N_{1} \geqslant \sup \left\{\left\|J_{\lambda}^{s}(p)\left(x_{\mu}(p, f)(s)\right)\right\| ; s \in T, 0<\lambda \leqslant 1\right\}
$$

Из неравенств (2.7), (3.9), (3.18), (4.19) следует, что

$$
\left|\varphi_{\lambda}^{t}(p)\left(x_{\mu}(p, f)(t)\right)\right| \leqslant \varphi_{\lambda}^{t}(p)\left(x_{\mu}(p, f)(t)\right)+2 \alpha\left(K+N_{1}\right)+2 \alpha .
$$

Поэтому

$$
\begin{aligned}
&\left|\varphi_{\lambda}^{t}(p)\left(x_{\mu}(p, f)(t)\right)\right|+\frac{1}{2} \int_{0}^{t}\left\|\dot{x}_{\mu}(p, f)(s)\right\|^{2} d s \\
& \leqslant R+\frac{1}{2} \int_{0}^{t}\|f(s)\|^{2} d s+\int_{0}^{t}\left(\left|\dot{a}_{r}(p)(s)\right|^{2}+\left|b_{r}(p)(s)\right|\right) \\
& \times\left(1+\left|\varphi_{\lambda}^{s}(p)\left(x_{\mu}(p, f)(s)\right)\right|\right) d s+2 \alpha\left(K+N_{1}\right)+2 \alpha, \quad \lambda, \mu \in(0,1] .
\end{aligned}
$$

Из неравенств (4.10), (4.22), свойства (4) предположения $H(\varphi)$ и неравенства Беллмана-Гронуолла вытекает, что

$$
\begin{gathered}
\left\|\dot{x}_{\mu}(p, f)\right\|_{L^{2}(T, H)} \leqslant N_{2}, \quad \mu \in(0,1] \\
\left\|\varphi_{\lambda}^{t}(p)\left(x_{\mu}(p, f)(t)\right)\right\| \leqslant N_{2}, \quad \lambda, \mu \in(0,1], \quad p \in \Lambda, \quad t \in T
\end{gathered}
$$

при некотором $N_{2}>0$. Теперь неравенства (4.13)-(4.15) вытекают из неравенств (4.19), (4.23), (4.24). Теорема доказана.

Теорема 4.2. Для любого $x(p, f) \in \mathscr{R}_{L}$ имеют место неравенства

$$
\begin{gathered}
\|x(p, f)(t)\| \leqslant N, \quad t \in T, \\
\|\dot{x}(p, f)\|_{L^{2}(T, H)} \leqslant N, \\
\left|\varphi^{t}(p)(x(p, f)(t))\right| \leqslant N, \quad t \in T .
\end{gathered}
$$

ДоказАТЕЛьство. Пусть $x(p, f) \in \mathscr{R}_{L}$. Тогда из результатов работы [9; $\S 1.4]$ следует, что

$$
\begin{aligned}
& x_{\lambda}(p, f) \rightarrow x(p, f) \quad \text { в } C(T, H) \quad \text { при } \quad \lambda \downarrow 0, \\
& \dot{x}_{\lambda}(p, f) \rightarrow \dot{x}(p, f) \quad \text { в } \quad L^{2}(T, H) \quad \text { при } \quad \lambda \downarrow 0 .
\end{aligned}
$$

Поэтому неравенства (4.25), (4.26) вытекают из (4.13), (4.14) и (4.28), (4.29). Воспользовавшись $(2.4),(2.9)$ и (4.28), мы получим

$$
\begin{gathered}
\lim _{\mu_{n} \downarrow 0} \varphi_{\lambda}^{t}(p)\left(x_{\mu_{n}}(p, f)(t)\right)=\varphi_{\lambda}^{t}(p)(x(p, f)(t)) \leqslant \varphi^{t}(p)(x(p, f)(t)) \\
\leqslant \frac{\lim }{\lambda_{n} \downarrow 0} \varphi_{\lambda_{n}}^{t}(p)\left(x_{\lambda_{n}}(p, f)(t)\right), \quad t \in T, \quad \lambda \in(0,1] .
\end{gathered}
$$

Теперь неравенство (4.27) следует из (4.15) и (4.30). Теорема доказана. 


\section{§ 5. Основные результаты}

Как уже отмечалось ранее, для любой функции $x(\cdot) \in L^{2}(T, H)$ функции $t \rightarrow \varphi^{t}(p)(x(t)), t \rightarrow \varphi_{\lambda}^{t}(p)(x(t)), t \rightarrow \partial \varphi_{\lambda}^{t}(p)(x(t))$ измеримы. Используя эти свойства и неравенство (3.9), мы можем определить функцию

$$
\Phi(p)(x)=\int_{T} \varphi^{t}(p)(x(t)) d t, \quad x(\cdot) \in L^{2}(T, H), \quad p \in \Lambda,
$$

которая является собственной выпуклой и полунепрерывной снизу.

Пусть $\Phi_{\lambda}(p), \lambda \in(0,1], p \in \Lambda,-$ регуляризация Моро-Иосиды функции $\Phi(p): L^{2}(T, H) \rightarrow(-\infty,+\infty]$. Тогда из лемм $1.2 .2,1.2 .4,1.5 .3$ и утверждения 0.3.3 из [9] вытекает следующая лемма.

Лемма 5.1. В рамках предположений $H(\varphi)$ справедливы следующие утверждения:

а) выполняется

$$
\Phi_{\lambda}(p)(x)=\int_{T} \varphi_{\lambda}^{t}(p)(x(t)) d t
$$

б) для любих $p \in \Lambda, \lambda \in(0,1]$

$$
\partial \Phi_{\lambda}(p)(x)(t)=\partial \varphi_{\lambda}^{t}(p)(x(t)) \text { n.в.; }
$$

в) если $f \in L^{2}(T, H)$, то $f \in \partial \Phi(p)(x)$ тогда и только тогда, когда

$$
f(t) \in \partial \varphi^{t}(p)(x(t)) \quad \text { n.в., } \quad p \in \Lambda .
$$

Теорема 5.1. Для любой последователъности $p_{n} \rightarrow p$ последователъность $\Phi\left(p_{n}\right), n \geqslant 1$, сходится $\kappa \Phi(p)$ в смысле Моско.

ДокАзАтЕЛьство. Из неравенств (2.7), (2.8) и (3.8) вытекает, что

$$
\begin{aligned}
&-\alpha-\alpha\left\|J_{\lambda}^{t}(p)(x)\right\| \leqslant \varphi_{\lambda}^{t}(p)(x) \leqslant \varphi_{\lambda}^{t}(p)\left(y^{t}(p)\right) \\
&+\left\|\partial \varphi_{\lambda}^{t}(p)(x)\right\|\left\|x-y^{t}(p)\right\|, \quad p \in \Lambda, \quad \lambda \in(0,1], \quad x \in H, \quad t \in T,
\end{aligned}
$$

где $y^{t}(p)$ - непрерывная функция, удовлетворяющая неравенству (3.17). Воспользовавшись этим неравенством и (3.18), (3.19), мы получим

$$
\begin{aligned}
-\alpha-\alpha & (K+\|x\|) \leqslant \varphi_{\lambda}^{t}(p)(x) \leqslant R+\frac{1}{\lambda}(K+2\|x\|) \\
+ & \frac{1}{\lambda}(K+2\|x\|) R, \quad p \in \Lambda, \quad \lambda \in(0,1], \quad x \in H .
\end{aligned}
$$

Пусть $x(\cdot) \in L^{2}(T, H), \lambda \in(0,1]$ произвольны и фиксированы. Тогда из (5.4) следует, что существует функция $g_{\lambda}(\cdot) \in L^{1}\left(T, \mathscr{R}^{*}\right)$ такая, что

$$
\left|\varphi_{\lambda}^{t}(p)(x(t))\right| \leqslant g_{\lambda}(t) \text { п.в., } \quad p \in \Lambda .
$$

Пусть $p_{n} \rightarrow p$. Так как $\varphi^{t}\left(p_{n}\right) \stackrel{M}{\longrightarrow} \varphi^{t}(p)$, то согласно теореме 3.26 из [2]

$$
\varphi_{\lambda}^{t}\left(p_{n}\right)(x(t)) \rightarrow \varphi_{\lambda}^{t}(p)(x(t)) \text { п.в. }
$$


Тогда из (5.2), (5.5) и теоремы Лебега об ограниченной сходимости вытекает, что

$$
\Phi_{\lambda}\left(p_{n}\right)(x) \rightarrow \Phi_{\lambda}(p)(x), \quad x \in L^{2}(T, H), \quad \lambda \in(0,1] .
$$

Поэтому согласно теореме 3.26 из [2] $\Phi\left(p_{n}\right) \stackrel{M}{\longrightarrow} \Phi(p)$. Теорема доказана.

Пусть $t \in(0,1]$. По аналогии с $(5.1)$ на пространстве $L^{2}([0, t], H)$ мы можем определить собственную выпуклую полунепрерывную снизу функцию

$$
\Phi^{t}(p)(x)=\int_{0}^{t} \varphi^{\tau}(p)(x(\tau)) d \tau, \quad x \in L^{2}([0, t], H) .
$$

СлеДСТВИЕ 5.1. Для любого $0<t \leqslant 1$ и любой последовательности $p_{n} \rightarrow p$, $n \geqslant 1$, последовательность $\Phi^{t}\left(p_{n}\right), n \geqslant 1$, сходится $\kappa \Phi^{t}(p)$ в смысле Моско.

Следствие непосредственно вытекает из теоремы 5.1.

ТЕОРема 5.2. Пусть $p_{n} \rightarrow p u f_{n} \rightarrow f$ в $\omega-L^{2}(T, H)$ и выполняется одно из условий:

(I) множество $\left\{f_{n}(t)\right\}_{n \geqslant 1}$ относителъно компактно в $H$ при почти каждом $t \in T$

(II) для любого $r>0$ любая последовательность $z_{n} \in H$, удовлетворяющая неравенствам $\left\|z_{n}\right\| \leqslant r,\left|\varphi^{t}\left(p_{n}\right)\left(z_{n}\right)\right| \leqslant r, n \geqslant 1, t \in T$, относительно компактна в $H$.

Тогда для последовательности $x\left(p_{n}, f_{n}\right), n \geqslant 1$, решений включения (4.8) справедливы утверждения

$$
\begin{gathered}
x\left(p_{n}, f_{n}\right) \rightarrow x(p, f) \quad \text { в } C(T, H) ; \\
\dot{x}\left(p_{n}, f_{n}\right) \rightarrow \dot{x}(p, f) \quad \text { в } \quad \omega-L^{2}(T, H) ; \\
\Phi^{t}\left(p_{n}\right)\left(x\left(p_{n}, f_{n}\right)\right) \rightarrow \Phi^{t}(p)(x(p, f))
\end{gathered}
$$

равномерно по $t \in T$, где $\Phi^{t}(p)(x)$ - функция, определенная равенством (5.6).

ДоказАтельство. Пусть выполняется условие (I). Тогда $f_{n} \in S_{L}$ при некотором $L>0$ (см. (4.10)). Поэтому $x\left(p_{n}, f_{n}\right) \in \mathscr{R}_{L}$ (см. (4.11)). Воспользовавшись (4.26) и неравенством Гёльдера, мы получаем

$$
\left\|x\left(p_{n}, f_{n}\right)(t)-x\left(p_{n}, f_{n}\right)(s)\right\| \leqslant|t-s|^{1 / 2} N .
$$

Получаем, что последовательность $x\left(p_{n}, f_{n}\right), n \geqslant 1$, равностепенно непрерывна в $C(T, H)$.

Пусть $B_{N}=\{z \in H ;\|z\| \leqslant N\}$. Тогда из (4.25) и теоремы 4 из [14] вытекает, что последовательность $x\left(p_{n}, f_{n}\right), n \geqslant 1$, относительно компактна в пространстве $\omega-C(T, H)$. Поэтому согласно (4.26) существует последовательность $n_{k}$, $k \geqslant 1$, такая, что

$$
\begin{aligned}
& x\left(p_{n_{k}}, f_{n_{k}}\right) \rightarrow x(\cdot) \quad \text { в } \quad \omega-C(T, H), \\
& \dot{x}\left(p_{n_{k}}, f_{n_{k}}\right) \rightarrow z(\cdot) \quad \text { в } \quad \omega-L^{2}(T, H) .
\end{aligned}
$$


В дальнейшем последовательности $x\left(p_{n_{k}}, f_{n_{k}}\right), \dot{x}\left(p_{n_{k}}, f_{n_{k}}\right), p_{n_{k}}, f_{n_{k}}$ мы будем обозначать $x_{k}, \dot{x}_{k}, p_{k}, f_{k}$.

Поскольку для любого $h \in H$ имеет место равенство

$$
\left\langle h, x_{k}(t)\right\rangle=\left\langle h, y^{0}\left(p_{k}\right)\right\rangle+\int_{0}^{t}\left\langle h, \dot{x}_{k}(s)\right\rangle d s, \quad t \in T, \quad k \geqslant 1,
$$

то из $(5.10),(5.11)$ и теоремы 3 из [14] следует, что

$$
\langle h, x(t)\rangle=\left\langle h, y^{0}(p)\right\rangle+\left\langle h, \int_{0}^{t} z(s) d s\right\rangle, \quad t \in T .
$$

Поэтому

$$
x(t)=y^{0}(p)+\int_{0}^{t} z(s) d s, \quad t \in T, \quad \text { и } \quad \dot{x}(t)=z(t) \text { п.в. }
$$

Из этих равенств следует, что $x(\cdot) \in W^{1,2}(T, H), x(0)=y^{0}(p)$ и

$$
\dot{x}_{k} \rightarrow \dot{x} \quad \text { в } \omega-L^{2}(T, H) .
$$

Покажем, что $x=x(p, f)$. Так как $x_{k} \rightarrow x$ в $\omega$ - $C(T, H)$ и пространство $C(T, H)$ непрерывно вложено в $L^{2}(T, H)$, то

$$
x_{k} \rightarrow x \quad \text { в } \omega-L^{2}(T, H) .
$$

Пусть $0<t \leqslant 1$, и обозначим через $x_{k}^{*}, x^{*}$ сужения функций $x_{k}$ и $x$ на отрезок $[0, t]$. Из определения субдифференциала выпуклой функции, определения решения включения (4.8), (5.6) и утверждения в) леммы 5.1 вытекает

$$
\int_{0}^{t}\left\langle-\dot{x}_{k}(s)-f_{k}(s), g(s)-x_{k}(s)\right\rangle d s \leqslant \Phi^{t}\left(p_{k}\right)(g)-\Phi^{t}(p)\left(x^{*}\right) \quad \forall g \in L^{2}([0, t], H) .
$$

Согласно следствию 5.1

$$
\Phi^{t}\left(p_{k}\right) \stackrel{M}{\longrightarrow} \Phi^{t}(p) .
$$

Поэтому из определения сходимости по Моско следует, что существует последовательность $g_{k} \in L^{2}([0, t], H), k \geqslant 1$, такая, что

$$
\begin{gathered}
g_{k} \rightarrow x^{*} \quad \text { в } L^{2}([0, t], H), \\
\Phi^{t}\left(p_{k}\right)\left(g_{k}\right) \rightarrow \Phi^{t}(p)\left(x^{*}\right) .
\end{gathered}
$$

Из $(4.27),(5.6),(5.13),(5.15)$ следует, что $\Phi^{t}(p)\left(x^{*}\right) \leqslant N$. Перепишем неравенство (5.14) в виде

$$
\begin{aligned}
\int_{0}^{t}\left\langle\dot{x}_{k}(s),\right. & x(s)\rangle d s \leqslant \Phi^{t}\left(p_{k}\right)\left(g_{k}\right)-\Phi^{t}\left(p_{k}\right)\left(x_{k}^{*}\right) \\
& +\int_{0}^{t}\left\langle\dot{x}_{k}(s), g_{k}(s)\right\rangle d s+\int_{0}^{t}\left\langle f_{k}(s), g_{k}(s)-x_{k}(s)\right\rangle d s .
\end{aligned}
$$


Покажем, что

$$
\lim _{k \rightarrow \infty} \int_{0}^{t}\left\langle f_{k}(s), g_{k}(s)-x_{k}(s)\right\rangle d s=0 .
$$

В соответствии с (5.16), не нарушая общности, мы можем считать, что $g_{k}(s) \rightarrow x(s)$ почти всюду на $[0, t]$. Тогда согласно (5.10) и теореме 3 из [14] при почти каждом $s \in[0, t]$ последовательность $g_{k}(s)-x_{k}(s), k \geqslant 1$, сходится в $\omega$ - $H$ к нулевому элементу пространства $H$. Поэтому последовательность числовых функций $h \rightarrow\left\langle h, g_{k}(s)-x_{k}(s)\right\rangle, h \in H, k \geqslant 1$, при почти каждом $s \in[0, t]$ равностепенно непрерывна. Поскольку на равностепенно непрерывном множестве топология поточечной сходимости совпадает с топологией равномерной сходимости на компактах из $H$ и множество $\left\{f_{n}(s)\right\}, n \geqslant 1$, относительно компактно в $H$, то

$$
\lim _{k \rightarrow \infty}\left\langle f_{k}(s), g_{k}(s)-x_{k}(s)\right\rangle=0 \text { п.в. на }[0, t] .
$$

Из неравенства

$$
\left|\left\langle f_{k}(s), g_{k}(s)-x_{k}(s)\right\rangle\right| \leqslant\left\|g_{k}(s)\right\|\left\|x_{k} f_{k}(s)\right\|+N\left\|f_{k}(s)\right\|
$$

следует, что

$$
\begin{aligned}
\int_{E}\left|\left\langle f_{k}(s), g_{k}(s)-x_{k}(s)\right\rangle\right| d s \\
\quad \leqslant\left(\int_{E}\left\|g_{k}(s)\right\|^{2} d s\right)^{1 / 2}\left(\int_{T}\left\|f_{k}(s)\right\|^{2} d s\right)^{1 / 2}+N \int_{E}\left\|f_{k}(s)\right\| d s
\end{aligned}
$$

для любого измеримого множества $E \subset[0, t]$. Тогда из $(5.21)$ и теоремы Данфорда-Петтиса (см. [12; теорема 4.21.2]) следует, что для любого $\varepsilon>0$ существует $\delta>0$ такое, что

$$
\int_{E}\left|\left\langle f_{k}(s), g_{k}(s)-x_{k}(s)\right\rangle\right| d s<\varepsilon
$$

для любого $k \geqslant 1$ и любого измеримого множества $E \subset[0, t]$ с мерой, не превышающей $\delta$. Поэтому из (5.20), (5.22) и утверждения 2.4 из [15] вытекает, что имеет место равенство (5.19).

Переходя к пределу при $k \rightarrow \infty$ в (5.18) и учитывая (5.12), (5.16), (5.17) и (5.19), мы получим

$$
\begin{aligned}
\varlimsup_{k \rightarrow \infty} \int_{0}^{t}\left\langle\dot{x}_{k}(s), x_{k}(s)\right\rangle d s \leqslant \int_{0}^{t} & \langle\dot{x}(s), x(s)\rangle d s \\
& +\Phi^{t}(p)\left(x^{*}\right)-\varliminf_{k \rightarrow \infty} \Phi^{t}\left(p_{k}\right)\left(x_{k}^{*}\right) .
\end{aligned}
$$

Теперь из (5.15), (5.23) вытекает

$$
\|x(t)\|^{2} \geqslant \varlimsup_{k \rightarrow \infty}\left\|x_{k}(t)\right\|^{2} .
$$

С другой стороны, согласно $(5.10) x_{k}(t) \rightarrow x(t)$ в $\omega-H$ и, следовательно,

$$
\|x(t)\|^{2} \leqslant \underline{\lim _{k \rightarrow \infty}}\left\|x_{k}(t)\right\|^{2} .
$$


Поэтому

$$
\|x(t)\|=\lim _{k \rightarrow \infty}\left\|x_{k}(t)\right\| .
$$

Из этого равенства и сходимости $x_{k}(t) \rightarrow x(t)$ в $\omega$ - $H$ мы получаем, что $x_{k}(t) \rightarrow$ $x(t)$ в $H$. Поскольку $t$ произвольно и последовательность $x_{k}(t), k \geqslant 1$, равностепенно непрерывна в $C(T, H)$, то $x_{k}(\cdot) \rightarrow x(\cdot)$ в $C(T, H)$. Так как $-\dot{x}_{k}(t)-$ $f_{k}(t) \in \partial \varphi^{t}\left(p_{k}\right)\left(x_{k}(t)\right)$ и почти всюду $-\dot{x}_{k}(\cdot)-f_{k}(\cdot) \in L^{2}(T, H)$, то согласно утверждению в) в лемме 5.1

$$
-\dot{x}_{k}(\cdot)-f_{k}(\cdot) \in \partial \Phi\left(p_{k}\right)\left(x_{k}\right) .
$$

Так как $x_{k}(\cdot) \rightarrow x(\cdot)$ в $L^{2}(T, H)$, то из сходимости $-\dot{x}_{k}(\cdot)-f_{k}(\cdot) \rightarrow-\dot{x}(\cdot)-f(\cdot)$ в $\omega-L^{2}(T, H),(5.25)$, утверждения 3.59 и теоремы 3.66 из [2] вытекает

$$
-\dot{x}(\cdot)-f(\cdot) \in \partial \Phi(p)(x) .
$$

Из этого включения и утверждения в) в лемме 5.1 следует, что

$$
-\dot{x}(t)-f(t) \in \partial \varphi^{t}(p)(x(t)) \text { п.в. }
$$

Следовательно, $\partial \varphi^{t}(p)(x(t)) \neq \varnothing$ почти всюду на $T$. Поэтому $x(t) \in \operatorname{dom} \partial \varphi^{t}(p)$ почти всюду на $T$. Из (4.27) и свойства (1) предположения $H(\varphi)$ вытекает, что $\varphi^{t}(p)(x(t)) \leqslant N, t \in T$. Поэтому $x(t) \in \operatorname{dom} \varphi^{t}(p), t \in T$.

Поскольку $x(\cdot) \in W^{1,2}(T, H), x(0)=y^{0}(p)$, то $x(\cdot)$ является решением включения (4.8). Так как включение (4.8) имеет единственное решение, то $x(\cdot)=$ $x(p, f)$.

При доказательстве мы считали, что последовательность $g_{k}(s) \rightarrow x_{k}(s)$, $k \geqslant 1$, почти всюду на $T$ сходится в $\omega-H$ к нулевому элементу пространства $H$. На самом деле этим свойством обладает некоторая подпоследовательность последовательности $g_{k}(s)-x_{k}(s)$. Поэтому мы доказали, что существует подпоследовательность $x\left(p_{n_{m}}, f_{n_{m}}\right), m \geqslant 1$, последовательности $x\left(p_{n}, f_{n}\right), n \geqslant 1$, такая, что

$$
\begin{gathered}
x\left(p_{n_{m}}, f_{n_{m}}\right) \rightarrow x(p, f) \quad \text { в } C(T, H), \\
\dot{x}\left(p_{n_{m}}, f_{n_{m}}\right) \rightarrow \dot{x}(p, f) \quad \text { в } \quad \omega-L^{2}(T, H) .
\end{gathered}
$$

Если мы предположим, что последовательность $x\left(p_{n}, f_{n}\right), n \geqslant 1$, не сходится к $x(p, f)$ в $C(T, H)$, то найдется подпоследовательность $x\left(p_{n_{k}}, f_{n_{k}}\right), k \geqslant 1$, последовательности $x\left(p_{n}, f_{n}\right), n \geqslant 1$, такая, что любая подпоследовательность последовательности $x\left(p_{n_{k}}, f_{n_{k}}\right), k \geqslant 1$, не сходится к $x(p, f)$. Повторяя рассуждения, приведенные выше, к последовательности $x\left(p_{n_{k}}, f_{n_{k}}\right), k \geqslant 1$, и учитывая, что включение (4.8) имеет единственное решение, мы придем к противоречию. Тем самым, при выполнении условия (I) соотношения (5.7), (5.8) доказаны.

Пусть выполняется условие (II). Тогда, повторяя рассуждения, использованные при рассмотрении случая (I), мы получим, что последовательность $x\left(p_{n}, f_{n}\right), n \geqslant 1$, равностепенно непрерывна в $C(T, H)$. Более того, из неравенств $(4.25),(4.27)$ вытекает, что для каждого $t \in T$ множество $\left\{x\left(p_{n}, f_{n}\right)(t)\right\}_{n \geqslant 1}$ 
относительно компактно в $H$. Поэтому последовательность $x\left(p_{n}, f_{n}\right), n \geqslant 1$, относительно компактна в $C(T, H)$. Используя неравенство (4.26), мы получим, что существует подпоследовательность $x\left(p_{n_{k}}, f_{n_{k}}\right), k \geqslant 1$, последовательности $x\left(p_{n}, f_{n}\right), n \geqslant 1$, такая, что

$$
\begin{gathered}
x\left(p_{n_{k}}, f_{n_{k}}\right) \rightarrow x(\cdot) \quad \text { в } \quad C(T, H), \\
\dot{x}\left(p_{n_{k}}, f_{n_{k}}\right) \rightarrow \dot{x}(\cdot) \quad \text { в } \quad \omega-L^{2}(T, H) .
\end{gathered}
$$

Поскольку последовательность $g_{k}(\cdot)-x_{k}(\cdot), k \geqslant 1$, сходится в $L^{2}(T, H)$ к нулевому элементу пространства $L^{2}(T, H)$, то неравенство $(5.19)$ имеет место.

В остальном мы следуем рассуждениям, использованным при рассмотрении предположения (I), и тем самым доказываем справедливость соотношений $(5.7),(5.8)$.

Заменяя в (5.18) последовательности $p_{k}=p_{n_{k}}, g_{k}=g_{n_{k}}, x_{k}^{*}=x_{n_{k}}, k \geqslant 1$, на последовательности $p_{n}, g_{n}, x_{n}^{*}, n \geqslant 1$, мы придем к неравенству

$$
\begin{gathered}
\Phi^{t}\left(p_{n}\right)\left(x_{n}^{*}\right) \leqslant \Phi^{t}\left(p_{n}\right)\left(g_{n}\right)+\int_{0}^{t}\left\langle\dot{x}_{n}(s), g_{n}(s)-x(s)\right\rangle d s \\
+\int_{0}^{t}\left\langle f_{n}(s), g_{n}(s)-x_{n}(s)\right\rangle d s .
\end{gathered}
$$

Из этого неравенства и (5.7), (5.8) следует, что

$$
\varlimsup_{n \rightarrow \infty} \Phi^{t}\left(p_{n}\right)\left(x_{n}^{*}\right) \leqslant \Phi^{t}(p)\left(x^{*}\right) .
$$

Тогда в соответствии со следствием 5.1 и определением сходимости по Моско мы получаем, что

$$
\lim _{n \rightarrow \infty} \Phi^{t}\left(p_{n}\right)\left(x_{n}^{*}\right)=\Phi^{t}(p)\left(x^{*}\right), \quad t \in T
$$

Равномерная сходимость

$$
\Phi^{t}\left(p_{n}\right)\left(x\left(p_{n}, f_{n}\right)\right)=\Phi^{t}\left(p_{n}\right)\left(x_{n}^{*}\right) \quad \text { к } \quad \Phi^{t}(p)(x(p, f))=\Phi^{t}(p)\left(x^{*}\right)
$$

вытекает из (5.28), (5.6) и (4.27). Теорема доказана.

СлеДСтвиЕ 5.2. Пусть $p_{n} \rightarrow p u f_{n} \rightarrow f$ в $L^{2}(T, H)$. Тогда справедливъ утверждения теоремы 5.2.

Поскольку $f_{n} \rightarrow f$ в $L^{2}(T, H)$, то существует подпоследовательность $f_{n_{m}}$, $m \geqslant 1$, последовательности $f_{n}$, сходящаяся почти всюду к $f$. Поэтому для почти каждого $t \in T$ множество $\left\{f_{n_{m}}(t) ; m \geqslant 1\right\}$ относительно компактно в $H$. Тогда из теоремы 5.2 следует, что для последовательности $x\left(p_{n_{m}}, f_{n_{m}}\right), m \geqslant 1$, имеют место соотношения (5.26), (5.27). Доказательство того, что для самой последовательности $x\left(p_{n}, f_{n}\right), n \geqslant 1$, справедливы соотношения (5.7)-(5.9), проводится с помощью аргументов, использованных при доказательстве теоремы 5.2 . 


\section{§ 6. Приложение}

Пусть $\mathbb{R}^{N}$ - конечномерное евклидово пространство с нормой $\|\cdot\|, \Omega \subset R^{N}-$ ограниченная область с регулярной (см. [16]) границей $\partial \Omega, \Gamma=T \times \partial \Omega, C(\bar{\Omega})-$ пространство непрерывных функций из $\Omega$ в $\mathbb{R}$, которые допускают непрерывное продолжение на замыкание $\Omega$.

Рассмотрим соболевское пространство $W_{0}^{1, p}(\Omega), N+1 \leqslant p<\infty$, с его топологически сопряженным $W^{-1, q}(\Omega), \frac{1}{p}+\frac{1}{q}=1$, и пространство $H=L^{2}(\Omega)$. Хорошо известно, что вложения $W_{0}^{1, p}(\Omega) \subset L^{2}(\Omega) \subset W^{-1, q}(\Omega)$ непрерывны, плотны и компактны. Обозначим через $\langle\langle\cdot, \cdot\rangle\rangle$ каноническую билинейную форму, устанавливающую двойственность между $W_{0}^{1, p}(\Omega)$ и $W^{-1, q}(\Omega)$, а через $\langle\cdot, \cdot\rangle-$ скалярное произведение в $H$. Как обычно (см. [16]), мы считаем, что сужения форм $\langle\langle\cdot, \cdot\rangle\rangle$ и $\langle\cdot, \cdot\rangle$ на $W_{0}^{1, p}(\Omega) \times H$ совпадают.

Пусть $K^{t} \subset \mathbb{R}, t \in T$, - замкнутое выпуклое множество, содержащее нуль.

ПРЕДПОЛОжЕниЕ $H\left(K^{t}\right)$. Существует функция $а(\cdot) \in W^{1,2}(T)$ такая, что для любых $0 \leqslant s \leqslant t \leqslant 1$ выполняется неравенство

$$
\sup _{u \in K^{s}} d\left(u, K^{t}\right) \leqslant|a(t)-a(s)|,
$$

где $d\left(u, K^{t}\right)$ - расстояние от точки $u \in K^{s}$ до множества $K^{t}$.

Положим

$$
\begin{gathered}
\mathscr{K}^{t}=\left\{u \in L^{2}(\Omega) ; u(x) \in K^{t} \text { п.в. на } \Omega\right\}, \\
\mathscr{K}_{\infty}=\left\{u \in W_{0}^{1, N+1}(\Omega) ;\|\nabla u(x)\| \leqslant 1 \text { п.в. на } \Omega\right\} .
\end{gathered}
$$

Для элемента $u \in \mathbb{R}(u(\cdot) \in H)$ обозначим через $\operatorname{pr}_{K^{t}} u\left(\operatorname{pr}_{\mathscr{K}^{t}} u(\cdot)\right)$ проекцию этого элемента на множество $K^{t}\left(\mathscr{K}^{t}\right)$.

Лемма 6.1. Пусть $u(\cdot) \in W_{0}^{1, p}(\Omega)$. Тогда для элемента $\operatorname{pr}_{\mathscr{K}^{t}} u(\cdot), t \in T$, имеют место соотношения

$$
\begin{gathered}
\operatorname{pr}_{\mathscr{K}^{t}} u(\cdot) \in W_{0}^{1, p}(\Omega), \\
\left\|\nabla\left(\operatorname{pr}_{\mathscr{K}^{t}} u\right)(x)\right\| \leqslant\|\nabla u(x)\| \text { n.в. на } \Omega .
\end{gathered}
$$

ДокАЗАТЕЛЬСтво. Хорошо известно (см. [17]), что для $u(\cdot) \in W_{0}^{1, p}(\Omega)$ имеет место равенство

$$
\left(\operatorname{pr}_{\mathscr{K}^{t}} u\right)(x)=\operatorname{pr}_{K^{t}} u(x) \text { п.в. на } \Omega .
$$

Поскольку функция $u(\cdot)$ на $\Omega$ имеет обобщенную производную $\frac{\partial u}{\partial x_{i}}, i=1, \ldots, N$, то существует эквивалентная ей на $\Omega$ функция $v(\cdot)$ такая, что она имеет по $x_{i}$ обычную производную почти всюду на $\Omega$, причем $v(\cdot)$ при почти всех фиксированных $x^{(i)}=\left(x_{1}, \ldots, x_{i-1}, x_{i+1}, \ldots, x_{N}\right) \in \Omega^{(i)}\left(\Omega^{(i)}-\right.$ ортогональная проекция $\Omega$ на гиперплоскость $\left.x_{i}=0\right)$ является абсолютно непрерывной функцией на любом замкнутом отрезке $[a, b]$ изменения переменной $x_{i}$, содержащемся в $\Omega$ (см. [18; п. 6.4]). 
Поэтому из неравенства

$$
\left|\operatorname{pr}_{K^{t}} u(x)-\operatorname{pr}_{K^{t}} u(y)\right| \leqslant|u(x)-u(y)|, \quad x, y \in \Omega,
$$

и равенства (6.6) следует, что функция $\nabla\left(\operatorname{pr}_{\mathscr{K}^{t}} u\right)$ определена почти всюду на $\Omega$ и почти всюду на $\Omega$ имеет место неравенство (6.5). Поэтому $\operatorname{pr}_{\mathscr{K}^{t}} u(\cdot) \in$ $W^{1, p}(\Omega)$. Так как $\Omega$ имеет регулярную границу и $W^{1, p}(\Omega) \subset C(\bar{\Omega})$ (см. [16; гл. II,теорема 1.2]), то $\left.u\right|_{\partial \Omega}=0$. Следовательно, $\left.\operatorname{pr}_{\mathscr{K}^{t}} u\right|_{\partial \Omega}=0$. Тогда из леммы 1.33 из [16; гл. II] вытекает, что $\operatorname{pr}_{\mathscr{K}^{t}} u(\cdot) \in W_{0}^{1, p}(\Omega)$. Лемма доказана.

Рассмотрим функцию $\varphi_{1}(p): L^{2}(\Omega) \rightarrow(-\infty,+\infty], N+1 \leqslant p<\infty$,

$$
\varphi_{1}(p)(u)= \begin{cases}\frac{1}{p} \int_{\Omega}\|\nabla u(x)\|^{p} d x, & u \in W_{0}^{1, p}(\Omega), \\ +\infty & \text { в противном случае }\end{cases}
$$

которая является выпуклой, собственной, полунепрерывной снизу.

Пусть $I\left(\mathscr{K}^{t}\right): L^{2}(\Omega) \rightarrow(-\infty,+\infty]-$ индикаторная функция множества $\mathscr{K}^{t}$, $t \in T$, т.е.

$$
I\left(\mathscr{K}^{t}\right)(u)= \begin{cases}0, & u \in \mathscr{K}^{t} \\ +\infty & \text { в противном случае. }\end{cases}
$$

Индикаторная функция $I\left(\mathscr{K}_{\infty}\right): L^{2}(\Omega) \rightarrow(-\infty,+\infty]$ множества $\mathscr{K}_{\infty}$ определяется аналогично. Так как $\mathscr{K}^{t}, t \in T$, является выпуклым замкнутым подмножеством пространства $L^{2}(\Omega)$, а $\mathscr{K}_{\infty}$ - выпуклым замкнутым ограниченным подмножеством пространства $W_{0}^{1, N+1}(\Omega)$ и, следовательно, выпуклым компактным подмножеством пространства $L^{2}(\Omega)$, то $I\left(\mathscr{K}^{t}\right), t \in T, I\left(\mathscr{K}_{\infty}\right)$ являются выпуклыми собственными полунепрерывными снизу функциями из $L^{2}(\Omega)$ в $(-\infty,+\infty]$.

Положим

$$
\begin{gathered}
\varphi^{t}(p)(u)=\varphi_{1}(p)(u)+I\left(\mathscr{K}^{t}\right)(u), \quad u \in H, \quad N+1 \leqslant p<\infty, \\
\varphi^{t}(\infty)(u)=I\left(\mathscr{K}_{\infty}\right)(u)+I\left(\mathscr{K}^{t}\right)(u), \quad u \in H, \quad t \in T .
\end{gathered}
$$

Используя одноточечную компактификацию Александрова полупрямой $[N+1$, $+\infty)$, мы можем рассмотреть компактное метрическое пространство $\Lambda=$ $[N+1,+\infty]$ параметра $p$ и семейство функций $\varphi^{t}(p) \in \Gamma_{0}(H), t \in T, p \in \Lambda$, определенных по формулам (6.8), (6.9).

Лемма 6.2. Семейство функиий $\varphi^{t}(p) \in \Gamma_{0}(H), t \in T, p \in \Lambda$, определенных по формулам (6.8), (6.9), обладает следующими свойствами:

(1) для любых $r>0, p \in \Lambda$ множество $\left\{u \in H ; \varphi^{0}(p)(u)<r\right\}$ не пусто;

(2) для любых $p \in \Lambda, s, t \in T, s \leqslant t, u \in \operatorname{dom} \varphi^{s}(p)$ найдется $u_{1} \in \operatorname{dom} \varphi^{t}(p)$, удовлетворяющее неравенствам

$$
\begin{gathered}
\left\|u_{1}-u\right\|_{H} \leqslant|a(t)-a(s)||\Omega|^{1 / 2}, \\
\varphi^{t}(p)\left(u_{1}\right) \leqslant \varphi^{s}(p)(u),
\end{gathered}
$$

где $|\Omega|$ - мера множества $\Omega$; 
(3) для любой последовательности $p_{n} \in \Lambda, p_{n} \rightarrow+\infty$, и любого $r>0$ любая последовательность $u_{n} \in H, n \geqslant 1$, удовлетворяющая неравенствам

$$
\left\|u_{n}\right\|_{H} \leqslant r, \quad \varphi^{t}\left(p_{n}\right)\left(u_{n}\right) \leqslant r, \quad n \geqslant 1
$$

относительно компактна в $H$.

ДоказАтельство. Так как нулевой элемент $\Theta$ пространства $H$ принадлежит множеству $\operatorname{dom} \varphi^{0}(p), p \in \Lambda$, и $\varphi^{0}(p)(\Theta)=0$, то утверждение (1) очевидно.

Пусть $p \in[N+1, \infty), s, t \in T, s \leqslant t$ и $u \in \operatorname{dom} \varphi^{s}(p)$. Очевидно, что $\operatorname{dom} \varphi^{s}(p)=W_{0}^{1, p}(\Omega) \cap \mathscr{K}^{s}$. Положим $u_{1}=\operatorname{pr}_{\mathscr{K}^{t}} u$. Воспользовавшись (6.4), (6.6) и (6.1), мы получим, что $u_{1} \in \operatorname{dom} \varphi^{t}(p)$ и

$$
\left\|u_{1}-u\right\|_{H} \leqslant\left(\int_{\Omega}|a(t)-a(s)|^{2} d x\right)^{1 / 2} .
$$

Теперь неравенство (6.10) вытекает из (6.13), а неравенство (6.11) следует из $(6.5)$ и $(6.7),(6.8)$.

Если $p=+\infty$, то $u \in \operatorname{dom} \varphi^{s}(\infty)=\mathscr{K}_{\infty} \cap \mathscr{K}^{s}$. Тогда для элемента $u_{1}=\operatorname{pr}_{\mathscr{K}^{t}} u$ будут иметь место неравенства (6.13), (6.5). Из этих неравенств и (6.3) будут вытекать неравенства (6.10), (6.11).

Пусть $p_{n} \in \Lambda, p_{n} \rightarrow+\infty, r>0$ и любая последовательность $u_{n} \in H, n \geqslant 1$, удовлетворяет неравенствам (6.12). Тогда из (6.7), (6.8) следует, что $u_{n} \in$ $W_{0}^{1, p_{n}}(\Omega), n \geqslant 1$, и

$$
\left\{\int_{\Omega}\left\|\nabla u_{n}(x)\right\|^{p_{n}} d x\right\}^{1 / p_{n}} \leqslant\left\{p_{n} \varphi^{t}\left(p_{n}\right)\left(u_{n}\right)\right\}^{1 / p_{n}} \leqslant\left(p_{n} r\right)^{1 / p_{n}} \rightarrow 1 \text { при } n \rightarrow \infty .
$$

Из этого неравенства вытекает, что существует $C>0$, при котором имеют место неравенства

$$
\left(\int_{\Omega}\left\|\nabla u_{n}(x)\right\|^{p_{n}} d x\right)^{1 / p_{n}} \leqslant C, \quad n \geqslant 1 .
$$

Поскольку $p_{n} \geqslant N+1, u_{n} \in W_{0}^{1, p_{n}}(\Omega) \subset W_{0}^{1, N+1}(\Omega), n \geqslant 1$, то

$$
\begin{aligned}
& \left(\int_{\Omega}\left\|\nabla u_{n}(x)\right\|^{N+1} d x\right)^{1 /(N+1)} \\
& \quad \leqslant\left(\int_{\Omega}\left\|\nabla u_{n}(x)\right\|^{p_{n}} d x\right)^{1 / p_{n}}|\Omega|^{1 /(N+1)-1 / p} \leqslant C(|\Omega|+1)^{1 /(N+1)}, \quad n \geqslant 1 .
\end{aligned}
$$

Из (6.14) вытекает, что последовательность $u_{n}, n \geqslant 1$, ограничена в $W_{0}^{1, N+1}(\Omega)$. Тогда из компактности вложения $W_{0}^{1, N+1}(\Omega) \hookrightarrow H$ следует, что последовательность $u_{n}, n \geqslant 1$, относительно компактна в $H$. Лемма доказана.

ЛЕмма 6.3. Для любого $t \in T$ и любой последовательности $p_{n} \in \Lambda$, $p_{n} \rightarrow+\infty$, последовательность $\varphi^{t}\left(p_{n}\right), n \geqslant 1$, сходится $\kappa \varphi^{t}(\infty)$ в смисле Моско. 
ДокАЗАтельство. Пусть $t \in T$ фиксировано и $p_{n} \rightarrow+\infty$. Вначале покажем, что для любого $u \in \operatorname{dom} \varphi^{t}(\infty)$ существует последовательность $u_{n} \in H$, $n \geqslant 1$, такая, что

$$
u_{n} \rightarrow u \quad \text { в } H \quad \text { и } \quad \varphi^{t}\left(p_{n}\right)\left(u_{n}\right) \rightarrow \varphi^{t}(\infty)(u) .
$$

Если $u \in \operatorname{dom} \varphi^{t}(\infty)=\mathscr{K}_{\infty} \cap \mathscr{K}^{t}$, то из (6.3) следует, что $u \in W_{0}^{1, p}(\Omega), p \geqslant N+1$. Поэтому $u \in \operatorname{dom} \varphi^{t}\left(p_{n}\right), n \geqslant 1$. Положим $u_{n}=u, n \geqslant 1$. Воспользовавшись $(6.3),(6.7)$ и $(6.8)$, мы получим

$0 \leqslant \varphi^{t}\left(p_{n}\right)\left(u_{n}\right)=\frac{1}{p_{n}} \int_{\Omega}\left\|\nabla u_{n}(x)\right\|^{p_{n}} d x \leqslant \frac{1}{p_{n}}|\Omega| \rightarrow 0=\varphi^{t}(\infty)(u)$ при $p_{n} \rightarrow+\infty$.

Тем самым, (6.15) доказано.

Теперь нам остается показать, что для любой последовательности $u_{n} \in H$, $n \geqslant 1$, слабо сходящейся в $H$ к $u$, имеет место неравенство

$$
\varphi^{t}(\infty)(u) \leqslant \underline{\lim }_{n \rightarrow \infty} \varphi^{t}\left(p_{n}\right)\left(u_{n}\right) .
$$

Если $\underline{\lim }_{n \rightarrow \infty} \varphi^{t}\left(p_{n}\right)\left(u_{n}\right)=+\infty$, то неравенство (6.16) очевидно. Предположим, что $\underline{\lim }_{n \rightarrow \infty} \varphi^{t}\left(p_{n}\right)\left(u_{n}\right)<+\infty$. Тогда существует подпоследовательность $\left\{n^{\prime}\right\}$ последовательности $\{n\}$ такая, что

$$
0 \leqslant \varphi^{t}\left(p_{n^{\prime}}\right)\left(u_{n^{\prime}}\right) \leqslant r \quad \forall n^{\prime}
$$

при некотором $r>0$. Из этого неравенства вытекает

$$
\begin{aligned}
& \left\{\int_{\Omega}\left\|\nabla u_{n^{\prime}}(x)\right\|^{p_{n^{\prime}}} d x\right\}^{1 / p_{n^{\prime}}} \leqslant\left\{p_{n^{\prime}} \varphi^{t}\left(p_{n^{\prime}}\right)\left(u_{n^{\prime}}\right)\right\}^{1 / p_{n^{\prime}}} \\
& \leqslant\left(p_{n^{\prime}} r\right)^{1 / p_{n^{\prime}}} \rightarrow 1 \text { при } n^{\prime} \rightarrow \infty .
\end{aligned}
$$

Для удобства обозначений мы будем писать $p$ и $u_{p}$ вместо $p_{n^{\prime}}$ и $u_{n^{\prime}}$. Согласно (6.17) существует $C>0$ такое, что

$$
\left(\int_{\Omega}\left\|\nabla u_{p}(x)\right\|^{p} d x\right)^{1 / p} \leqslant C .
$$

Из этого неравенства непосредственно вытекает

$$
\begin{gathered}
\left(\int_{\Omega}\left\|\nabla u_{p}(x)\right\|^{q} d x\right)^{1 / q} \leqslant\left(\int_{\Omega}\left\|\nabla u_{p}(x)\right\|^{p} d x\right)^{1 / p}|\Omega|^{(p-q) /(p q)} \\
\leqslant C(|\Omega|+1)^{1 / q} \quad \forall q \in[N+1, p] .
\end{gathered}
$$

Воспользовавшись (6.19), мы получим, что для любого $q \in[N+1,+\infty)$ существует подпоследовательность $\left\{p_{q}\right\}$ последовательности $\{p\}$ такая, что последовательность $u_{p_{q}}$ слабо сходится к $u$ в пространстве $W_{0}^{1, q}(\Omega)$ при $p_{q} \rightarrow+\infty$. Так как $u_{p_{q}}$ слабо сходится к $u$ в $H$ при $p_{q} \rightarrow+\infty$, то $u_{p_{q}}$ слабо сходится к $u$ в пространстве $W_{0}^{1, q}(\Omega)$. Поэтому $u \in W_{0}^{1, N+1}(\Omega) \cap \mathscr{K}^{t}$ и

$$
\nabla u_{p_{q}} \rightarrow \nabla u \text { слабо в } L^{q}\left(\Omega, \mathbb{R}^{N}\right) .
$$


Для удобства в дальнейшем мы опускаем индекс $q$ в $p_{q}$. Тогда из $(6.17),(6.20)$ вытекает

$$
\begin{gathered}
\left\{\int_{\Omega}\|\nabla u(x)\|^{q} d x\right\}^{1 / q} \leqslant \underline{\lim _{p \rightarrow+\infty}}\left\{\int_{\Omega}\left\|\nabla u_{p}(x)\right\|^{q} d x\right\}^{1 / q} \\
\leqslant \lim _{p \rightarrow+\infty}\left\{\int_{\Omega}\left\|\nabla u_{p}(x)\right\|^{p} d x\right\}^{1 / p}|\Omega|^{(p-q) /(p q)} \\
\leqslant \lim _{p \rightarrow+\infty}(r p)^{1 / p}|\Omega|^{(p-q) /(p q)}=|\Omega|^{1 / q} .
\end{gathered}
$$

Переходя к пределу при $q \rightarrow+\infty$ в последнем неравенстве, мы получим

$$
\|\nabla u(x)\| \leqslant 1 \text { п.в. на } \Omega \text {. }
$$

Поэтому $u \in \mathscr{K}_{\infty} \cap \mathscr{K}^{t}$. Последнее означает, что $\varphi^{t}(\infty)(u)=0$. Тем самым, неравенство (6.16) имеет место.

Если $u \notin \operatorname{dom} \varphi^{t}(\infty)$, то из (6.16) следует, что для любой последовательности $u_{n} \rightarrow u$ в $H$ будет иметь место равенство $\lim _{n \rightarrow \infty} \varphi^{t}\left(p_{n}\right)\left(u_{n}\right)=\varphi^{t}(\infty)(u)$. Поэтому последовательность $\varphi^{t}\left(p_{n}\right), n \geqslant 1$, сходится в смысле Моско к $\varphi^{t}(\infty)$ для любого $t \in T$ и любой последовательности $p_{n} \in \Lambda, p_{n} \rightarrow+\infty$. Лемма доказана.

При доказательстве леммы 6.3 мы в основном следовали схеме доказательства леммы 3.6 из [6], где рассматривалось подобное утверждение.

Лемма 6.4. Для любого $t \in T$

$$
\begin{gathered}
\partial \varphi^{t}(p)=\partial \varphi_{1}(p)+\partial I\left(\mathscr{K}^{t}\right), \quad p \in[N+1,+\infty), \\
\partial \varphi^{t}(\infty)=\partial I\left(\mathscr{K}_{\infty}\right)+\partial I\left(\mathscr{K}^{t}\right) .
\end{gathered}
$$

ДокАЗАтЕльство. Включения

$$
\begin{gathered}
\partial \varphi_{1}(p)+\partial I\left(\mathscr{K}^{t}\right) \subset \partial \varphi^{t}(p), \quad p \in[N+1,+\infty), \\
\partial I\left(\mathscr{K}_{\infty}\right)+\partial I\left(\mathscr{K}^{t}\right) \subset \partial \varphi^{t}(\infty)
\end{gathered}
$$

хорошо известны. Поэтому для доказательства равенств (6.21), (6.22) нам достаточно доказать максимальную монотонность операторов

$$
\begin{gathered}
\partial \varphi_{1}(p)+\partial I\left(\mathscr{K}^{t}\right), \quad p \in[N+1,+\infty), \\
\partial I\left(\mathscr{K}_{\infty}\right)+\partial I\left(\mathscr{K}^{t}\right) .
\end{gathered}
$$

Пусть $\partial I_{\lambda}\left(\mathscr{K}^{t}\right)(u), u \in H, \lambda>0,-$ субдифференциал регуляризации МороИосиды функции $I\left(\mathscr{K}^{t}\right)(u)$. Известно (см. [2; 3.4 .3$\left.]\right)$, что

$$
\partial I_{\lambda}\left(\mathscr{K}^{t}\right)(u)=\frac{1}{\lambda}\left(u-\operatorname{pr}_{\mathscr{K}^{t}} u\right) .
$$

Пусть $u \in \operatorname{dom} \partial \varphi_{1}(p), v \in \partial \varphi_{1}(p)(u)$. Тогда $u \in W_{0}^{1, p}(\Omega)$ и согласно (6.4) $\operatorname{pr}_{\mathscr{K}^{t}} u \in W_{0}^{1, p}(\Omega)$. Воспользовавшись определением субдифференциала выпуклой функции и (6.23), мы получим

$$
\varphi_{1}(p)(u)-\varphi_{1}(p)\left(\operatorname{pr}_{\mathscr{K}^{t}} u\right) \leqslant\left\langle v, u-\operatorname{pr}_{\mathscr{K}^{t}} u\right\rangle=\lambda\left\langle v, \partial I_{\lambda}\left(\mathscr{K}^{t}\right)(u)\right\rangle .
$$


Теперь из (6.5), (6.7) и (6.24) вытекает, что

$$
\left\langle v, \partial I_{\lambda}\left(\mathscr{K}^{t}\right)(u)\right\rangle \geqslant 0 \quad \forall v \in \partial \varphi_{1}(p)(u), \quad u \in \operatorname{dom} \partial \varphi_{1}(p), \quad \lambda>0 .
$$

Отсюда и из теоремы 1.10 из [19] вытекает, что оператор $\partial \varphi_{1}(p)+\partial I\left(\mathscr{K}^{t}\right)$, $p \in[N+1,+\infty)$, максимально монотонен. Поэтому равенство (6.19) доказано. Справедливость равенства (6.20) доказывается аналогично. Лемма доказана.

Пусть $r>0$ и $y_{\infty} \in \operatorname{dom} \varphi^{0}(\infty), \varphi^{0}(\infty)\left(y_{\infty}\right) \leqslant r$. Тогда из лемм $6.2,6.3$ и следствия 3.1 вытекает, что для любой последовательности $p_{n} \rightarrow+\infty, n \geqslant 1$, существует последовательность $y_{n}, n \geqslant 1$, такая, что $y_{n} \rightarrow y_{\infty}$ в $L^{2}(\Omega)$ и $\varphi\left(p_{n}\right)\left(y_{n}\right) \leqslant r, n \geqslant 1$. Положим $z_{n}=z\left(p_{n}\right)$ и рассмотрим включения

$$
\begin{gathered}
-\dot{z}_{n}(t) \in \partial \varphi_{1}\left(p_{n}\right)\left(z_{n}(t)\right)+\partial I\left(\mathscr{K}^{t}\right)\left(z_{n}(t)\right)+f_{n}(t) \text { п.в., } \\
z_{n}(0)=y_{n}, \quad f_{n} \in L^{2}\left(T, L^{2}(\Omega)\right), \quad p_{n} \geqslant N+1 ; \\
-\dot{z}_{\infty}(t) \in \partial I\left(\mathscr{K}_{\infty}\right)\left(z_{\infty}(t)\right)+\partial I\left(\mathscr{K}^{t}\right)\left(z_{\infty}(t)\right)+f_{\infty}(t) \text { п.в. }, \\
z_{\infty}(0)=y_{\infty}, \quad f_{\infty} \in L^{2}\left(T, L^{2}(\Omega)\right) .
\end{gathered}
$$

Под решением включений (6.25), (6.26) понимаются функции $z_{n}(\cdot), z_{\infty}(\cdot) \in$ $W^{1,2}\left(T, L^{2}(\Omega)\right), z_{n}(0)=y_{n}, z_{\infty}(0)=y_{\infty}, n \geqslant 1$, такие, что $z_{n}(t) \in \operatorname{dom} \partial \varphi^{t}\left(p_{n}\right)$, $z_{\infty}(t) \in \operatorname{dom} \partial \varphi^{t}(\infty)$ почти всюду и почти всюду на $T$ выполняются включения (6.25), (6.26).

Теорема 6.1. Пусть $p_{n} \rightarrow+\infty$. Тогда для любого $n \geqslant 1$ каждое из включений $(6.25),(6.26)$ имеет и причем единственное решение $z_{n}(\cdot), z_{\infty}(\cdot)$ соответственно, причем $z_{n}(t) \in W_{0}^{1, p_{n}}(\Omega) \cap \mathscr{K}^{t} u z_{\infty}(t) \in \mathscr{K}_{\infty} \cap \mathscr{K}^{t}, t \in T$.

Если последовательность $f_{n}, n \geqslant 1$, слабо сходится в $L^{2}\left(T, L^{2}(\Omega)\right) \kappa f_{\infty}$, mo

$$
\begin{gathered}
z_{n}(\cdot) \rightarrow z_{\infty}(\cdot) \quad \text { в } C(T, H), \quad \dot{z}_{n}(\cdot) \rightarrow \dot{z}_{\infty}(\cdot) \quad \text { в } \omega-L^{2}\left(T, L^{2}(\Omega)\right), \\
\frac{1}{p_{n}} \int_{T}\left\|\nabla z_{n}(t)\right\|^{p_{n}} d t \rightarrow 0 .
\end{gathered}
$$

ДокАЗАТЕльство. Из лемм 6.2, 6.3 следует, что для функций $\varphi^{t}\left(p_{n}\right), \varphi^{t}(\infty)$, определенных равенствами (6.8), (6.9), выполняются все предположения, при которых имеет место теорема 5.2. Если воспользоваться равенствами (6.21), (6.22), то утверждения теоремы 6.1 вытекают из теоремы 5.2.

Пусть $I\left(K^{t}\right): \mathbb{R} \rightarrow(-\infty,+\infty]$ - индикаторная функция множества $K^{t} \subset \mathbb{R}$, $t \in T$, и $\partial I\left(K^{t}\right)$ - ее субдифференциал.

Через $\Delta_{p} u, p \geqslant N+1$, обозначим $p$-лапласиан

$$
\Delta_{p} u=\operatorname{div}\left(\|\nabla u\|^{p-2} \nabla u\right)
$$

Как обычно, мы отождествляем пространства $L^{2}\left(T, L^{2}(\Omega)\right)$ и $L^{2}(T \times \Omega)$, ставя в соответствие элементам $z \in L^{2}\left(T, L^{2}(\Omega)\right)$ элементы из $L^{2}(T \times \Omega)$ по правилу $z(t)(x)=z(t, x)$. 
Пусть $p_{n} \rightarrow+\infty$. Рассмотрим включения

$$
\begin{gathered}
-\frac{\partial z_{n}(t, x)}{\partial t} \in-\Delta_{p_{n}} z_{n}(t, x)+\partial I\left(K^{t}\right)\left(z_{n}(t, x)\right)+f_{n}(t, x), \\
f_{n} \in L^{2}(T \times \Omega), \\
z_{n}(0, x)=y_{n}(x), \quad y_{n}(x) \in K^{0} \text { п.в. на } \Omega, \\
y_{n} \in W_{0}^{1, p_{n}},\left.\quad z_{n}\right|_{\Gamma}=0 .
\end{gathered}
$$

При фиксированном $n \geqslant 1$ под решением включения (6.30) мы понимаем функцию $z_{n} \in W^{1,2}\left(T, L^{2}(\Omega)\right), z_{n}(0)(x)=y_{n}(x)$, такую, что $\Delta_{p_{n}} z_{n}(t, x)$ при почти каждом $t \in T$ является элементом пространства $L^{2}(\Omega)$ и почти всюду на $T$ при почти каждом $x \in \Omega$ будет иметь место включение (6.30).

Пусть оператор $A(p)$ из $W_{0}^{1, p}(\Omega)$ в $W^{-1, q}(\Omega)$, где $\frac{1}{p}+\frac{1}{q}=1, N+1 \leqslant p<+\infty$, определен по формуле

$$
\langle\langle A(p) u, v\rangle\rangle=\int_{\Omega}\left\langle\|\nabla u(x)\|^{p-2} \nabla u(x), \nabla v(x)\right\rangle d x, \quad u, v \in W_{0}^{1, p}(\Omega) .
$$

Известно (см. [20]), что оператор $A(p)$ является дифференциалом Гато функции

$$
\psi(p)(u)=\frac{1}{p} \int_{\Omega}\|\nabla u(x)\|^{p} d x, \quad u \in W_{0}^{1,2}(\Omega),
$$

и

$$
A(p) u=-\operatorname{div}\left(\|\nabla z\|^{p-2} \nabla u\right)=-\Delta_{p} u .
$$

Если рассмотреть сужение $A(p)$ на множество

$$
D=\left\{u \in W_{0}^{1, p}(\Omega) ; A u \in L^{2}(\Omega)\right\}
$$

то $A(p): D \subset L^{2}(\Omega) \rightarrow L^{2}(\Omega)$ является максимально монотонным оператором (см. [20]) и

$$
-\Delta_{p} u=\partial \varphi_{1}(p)(u)
$$

где $\varphi_{1}(p)$ - функция, определенная по формуле (6.7).

Пусть $z \in L^{2}(\Omega), y \in L^{2}(\Omega)$. Хорошо известно, что следующие условия эквивалентны:

$$
\begin{gathered}
y \in \partial I\left(\mathscr{K}^{t}\right)(z), \\
y(x) \in \partial I\left(K^{t}\right)(z(x)) \text { п.в. на } \Omega .
\end{gathered}
$$

ТеОрема 6.2. Любое решение включения (6.25) является решением включения (6.30). Справедливо и обратное утверждение.

ДоказАтельство. Пусть $z_{n}, n \geqslant 1$, является решением включения (6.25). Тогда $z_{n} \in W^{1,2}\left(T, L^{2}(\Omega)\right)$ и имеют место соотношения (6.31), (6.32). Из определения решения включения (6.25) вытекает, что $z_{n}(t) \in \operatorname{dom} \partial \varphi_{1}^{t}\left(p_{n}\right)$ при почти каждом $t \in T$. Поэтому из $(6.33)$ вытекает, что $\Delta_{p_{n}} z_{n}(t)$ при почти каждом $t \in T$ является элементом пространства $L^{2}(\Omega)$. Поэтому

$$
-\dot{z}(t)+\Delta_{p_{n}} z_{n}(t)-f_{n}(t) \in L^{2}(\Omega) .
$$


Из этого включения, (6.25), (6.34), (6.35) вытекает, что включение (6.30) выполняется почти всюду на $T$ при почти каждом $x \in \Omega$. Тем самым, $z_{n}, n \geqslant 1$, является решением включения (6.30).

Пусть $z_{n}, n \geqslant 1,-$ решение включения (6.30). Тогда $z_{n} \in W^{1,2}\left(T, L^{2}(\Omega)\right)$, $z_{n}(0)=y_{n}$, и при почти каждом $t \in T$ функция $\Delta_{p_{n}} z_{n}(t, x)$ является элементом пространства $L^{2}(\Omega)$. Поэтому при почти каждом $t \in T$

$$
-\dot{z}_{n}(t, x)+\Delta_{p_{n}} z_{n}(t, x)-f_{n}(t, x)
$$

является элементом пространства $L^{2}(\Omega)$ и

$$
-\dot{z}_{n}(t, x)+\Delta_{p_{n}} z_{n}(t, x)-f_{n}(t, x) \in \partial I\left(K^{t}\right)\left(z_{n}(t, x)\right) .
$$

Из этого включения и $(6.34),(6.35)$ вытекает, что почти всюду на $T$

$$
-\dot{z}_{n}(t)+\Delta_{p_{n}} z_{n}(t)-f_{n}(t) \in \partial I\left(\mathscr{K}^{t}\right)\left(z_{n}(t)\right) .
$$

Воспользовавшись $(6.36),(6.33),(6.8)$, получаем, что почти всюду на $T$ имеет место включение $(6.25)$ и $z_{n}(t) \in \operatorname{dom} \partial \varphi^{t}\left(p_{n}\right)$ почти всюду. Поэтому $z_{n}, n \geqslant 1$, является решением включения (6.25). Теорема доказана.

Пусть $f_{n} \in L^{2}(T \times \Omega), n \geqslant 1$, слабо сходится в $L^{2}(T, \Omega)$ к $f_{\infty}$. Воспользовавшись теоремами $6.1,6.2$, мы можем сказать, что пределом последовательности решений $z_{n}, n \geqslant 1$, включения (6.30) при $p_{n} \rightarrow+\infty$ является решение включения (6.26). Тем самым, мы дали ответ на вопрос, поставленный в 1 .

\section{Список литературы}

[1] H. Brézis, Opérateurs maximaux monotones et semi-groupes de contractions dans les espaces de Hilbert, North-Holland, Amsterdam-London; Elsevier, New York, 1973.

[2] H. Attouch, Variational convergence for functions and operators, Appl. Math. Ser., Pitman, Boston-London-Melbourne, 1984.

[3] G. Aronsson, L. C. Evans, Y. Wu, "Fast/slow diffusion and growing sandpiles", J. Differential Equations, 131:2 (1996), 304-335.

[4] J. W. Barrett, L. Prigozhin, "Bean's critical-state model as the $p \rightarrow \infty$ limit of an evolutionary p-Laplacian equation", Nonlinear Anal., 42:2 (2000), 977-993.

[5] H.-M. Yin, "On a $p$-Laplacian type of evolution system and applications to the Bean model in the type-II superconductivity theory", Quart. Appl. Math., 59:1 (2001), 47-66.

[6] G. Akagi, "Convergence of functionals and its applications to parabolic equations", Abstr. Appl. Anal., 11 (2004), 907-933.

[7] G. Akagi, M. Otani, "Time-dependent constraint problems arising from macroscopic critical-state models for type-II superconductivity and their approximations", Adv. Math. Sci. Appl., 14:2 (2004), 683-712.

[8] P. Krejčí, J. Sprekels, "Parabolic regularization of differential inclusions and the stop operator", Interfaces Free Bound., 4:4 (2004), 423-435.

[9] N. Kenmochi, "Solvability of nonlinear evolution equations with time-dependent constraints and applications", Bull. Fac. Educ., Chiba Univ., 2, 30 (1981), 1-87.

[10] Н. Бурбаки, Топологические векторные пространства, Элементы математики, ИЛ, М., 1959; пер. с фр.: N. Bourbaki, Éléments de mathématique. XIX. Part 1: Les structures fondamentales de l'analyse. Livre V: Espaces vectoriels topologiques, Hermann, Paris, 1955. 
[11] E. Michael, "Continuous Selections. I", Ann. of Math. (2), 63:2 (1956), 361-382.

[12] Р. Эдвардс, Функииональный анализ. Теория и приложения, Мир, М., 1969; пер. с англ.: R. E. Edwards, Functional analysis. Theory and applications, Holt, Rinehart and Winston, New York-Toronto-London, 1965.

[13] N. Kenmochi, "On the quasi-linear heat equation with time-dependent obstacles", Nonlinear Anal., 5:1 (1981), 71-80.

[14] A. A. Tolstonogov, D. A. Tolstonogov, " $L_{p}$-continuous extreme selectors of multifunctions with decomposable values: existence theorems", Set-Valued Anal., 4:2 (1996), 173-203.

[15] M. Kisielewicz, "Weak compactness in spaces $C(S, X)$ ", Information theory, statistical decision functions, random processes, Trans. 11th Prague Conf. (Prague, 1990), Kluwer Acad. Publ., Dordrecht, 1992, 101-106.

[16] Х. Гаевский, К. Грегер, К. Захариас, Нелинейные операторные уравнения и операторные дифференциальные уравнения, Мир, М., 1978; пер. с нем.: Н. Gajewski, K. Gröger, K. Zacharias, Nichtlineare Operatorgleichungen und Operatordifferentialgleichungen, Akademie-Verlag, Berlin, 1974.

[17] F. Hiai, H. Umegaki, "Integrals, conditional expectations, and martingales of multivalued functions", J. Multivariate Anal., 7:1 (1977), 149-182.

[18] О.В.Бесов, В. П. Ильин, С. М. Никольский, Интегралъные представления функиий и теоремъ вложения, 2-е изд., Наука, М., 1996; англ. пер. 1-го изд.: O. V. Besov, V.P. Il'in, S. M. Nikol'skiŭ, Integral representations of functions and imbedding theorems, vol. I, II, Winston, Washington, DC; Wiley, New York-Toronto, ON-London, 1979.

[19] V. Barbu, Optimal control of variational inequalities, Res. Notes in Math., 100, Pitman, Boston-London-Melbourne, 1984.

[20] V. Barbu, Nonlinear semigroups and differential equations in Banach spaces, Noordhoff, Leyden, 1976.

\author{
А. А. Толстоногов (А. А. Tolstonogov) \\ Институт динамики систем \\ и теории управления СО РАН, г. Иркутск \\ E-mail: aatol@icc.ru
}

Поступила в редакцию 26.03.2008 и 03.12 .2008 